\title{
Asymmetric Allylboration of Cyclic Imines and Applications to
}

\author{
Alkaloid Synthesis \\ T. Robert Wu and J. Michael Chong* \\ Guelph-Waterloo Centre for Graduate Work in Chemistry and \\ Biochemistry, Department of Chemistry, University of Waterloo, Waterloo, \\ Ontario, Canada N2L $3 G 1$
}

Supporting Information

Table of Contents

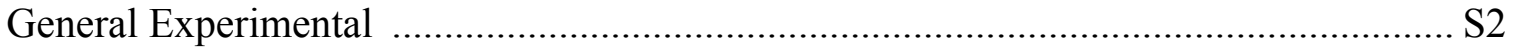

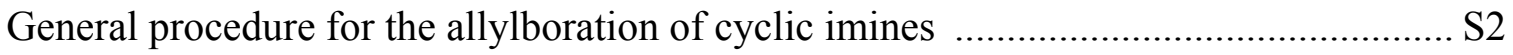

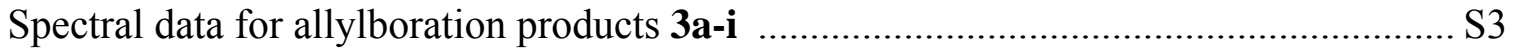

Preparation and spectral data of $(+)$-crispine A (4) ............................................ S12

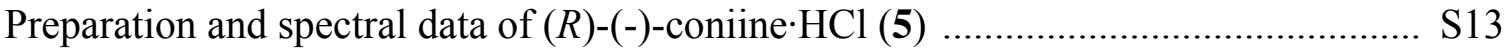

Preparation and spectral data of compound 6-8 .................................................. S13

Preparation and spectral data of ent-corynantheidol (9) ....................................... S16

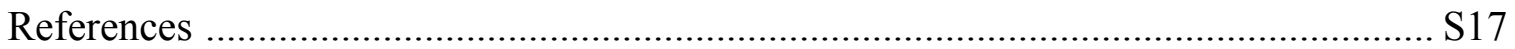

${ }^{1} \mathrm{H}$ and ${ }^{13} \mathrm{C}$ NMR spectra for compounds 3c-e, 3g, 4, 5, 7-9 ............................... S19 


\section{General Experimental}

All reactions were performed using flame-dried glassware under an argon atmosphere. Dichloromethane was freshly distilled from calcium hydride. Tetrahydrofuran, diethyl ether, and toluene were freshly distilled from sodium/benzophenone. Chiral 3,3'-disubstituted binaphthols were synthesized using procedures from a previous report. ${ }^{1}$ Cyclic imines were prepared according to literature procedures. ${ }^{2}{ }^{1} \mathrm{H}$ and ${ }^{13} \mathrm{C}$ NMR spectra were recorded in $\mathrm{CDCl}_{3}$ at $300 \mathrm{MHz}$ and $75 \mathrm{MHz}$, respectively. Mass spectra were recorded on a Kratos MA890 mass spectrometer using electron impact (EI, $70 \mathrm{ev)}$ ionization unless otherwise specified. Optical rotations were recorded in cells with $10 \mathrm{~cm}$ path length on a Perkin-Elmer 241 digital polarimeter.

\section{General procedure for the allylboration of cyclic imines:}

To a solution of the appropriate $3,3^{\prime}$-disubstitutedbinaphthol $(0.35 \mathrm{mmol})$ in THF (5

$\mathrm{mL}$ ) was added triallylborane ${ }^{1}(46 \mathrm{mg}, 0.34 \mathrm{mmol})$ dropwise. The reaction was stirred at room temperature for 2 hours then brought to reflux for 1 hour ( 2 hours for $\mathbf{2 e - h}$ ). The allylboronate solution was cooled to room temperature and concentrated under reduced pressure. The resulting white solid was dissolved in toluene $(5 \mathrm{~mL})$ and THF $(1 \mathrm{~mL})$ and the solution was cooled to $-78{ }^{\circ} \mathrm{C}$. A solution of the imine $(0.21 \mathrm{mmol}, 0.1-0.5 \mathrm{M})$ in THF was added dropwise over 2 minutes. The reaction was stirred at $-78{ }^{\circ} \mathrm{C}$ for 24 hours and warmed to room temperature for an additional 24 hours. $\mathrm{MeOH}(5 \mathrm{~mL})$ and aqueous $\mathrm{NH}_{4} \mathrm{Cl}$ were used to quench the reaction. The organic phase was washed with brine and dried over $\mathrm{Na}_{2} \mathrm{SO}_{4}$. Purification using flash column chromatography on silica gel 
$\left(\mathrm{EtOAc} / \mathrm{MeOH} / \mathrm{NH}_{4} \mathrm{OH}\right)$ gave the desired homoallylic amine and the chiral ligand in their pure forms.

Alternatively, the reaction mixture could be extracted with $2 \mathrm{M} \mathrm{HCl}(5 \times 5 \mathrm{~mL})$. The chiral ligand was recovered from the organic phase. The aqueous phase was then basified using $1 \mathrm{M} \mathrm{NaOH}$ and extracted with diethyl ether $(5 \times 5 \mathrm{~mL})$. The combined ethereal extracts were dried over $\mathrm{Na}_{2} \mathrm{SO}_{4}$ and evaporation of the solvent gave the homoallylic amine.

The enantiomeric purities of the products were determined by HPLC analysis ( $4.6 \mathrm{x}$ $250 \mathrm{~mm}$ ChiralCel OD, hexane $/ i-\mathrm{PrOH}=99.5 / 0.5 \sim 98 / 2)$ of their trifluoroacetamides unless otherwise specified.

\section{(R)-1-Allyl-1,2,3,4-tetrahydroisoquinoline (3a).}

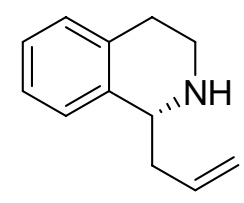

$[\alpha]_{589}^{25}+91$ (95\% ee, c 0.51, THF) [lit. ${ }^{3}\left(S\right.$ enantiomer): $[\alpha]^{25}{ }_{589}-98.1$ (94.8\% ee, c 0.93, THF)]; IR (neat): 3304, 3073, 3018, 1639,1583, 913, $742 \mathrm{~cm}^{-1}$; ${ }^{1} \mathrm{H}$ NMR (300 MHz, $\left.\mathrm{CDCl}_{3}\right): \delta 1.92(\mathrm{~s}, \mathrm{br}, 1 \mathrm{H}), 2.43-3.02(\mathrm{~m}, 5 \mathrm{H}), 3.22(\mathrm{dt}, J=11.1 \mathrm{~Hz}, 5.4 \mathrm{~Hz}, 1 \mathrm{H}), 4.03(\mathrm{dd}$, $J=3.3 \mathrm{~Hz}, 9.0 \mathrm{~Hz}, 1 \mathrm{H}), 5.09-5.22(\mathrm{~m}, 2 \mathrm{H}), 5.83(\mathrm{~m}, 1 \mathrm{H}), 7.04-7.17$ (m, 4H); ${ }^{13} \mathrm{C} \mathrm{NMR}$ (75 MHz, $\left.\mathrm{CDCl}_{3}\right): \delta 138.6,135.5,135.3,129.2,125.9,125.9,125.7,117.8,55.0,41.0$, 40.5, 29.9; Enantiomeric excess was determined by HPLC analysis of its trifluoroacetamide: (hexane $/ i-\mathrm{PrOH}=99 / 1$, flow rate $=1 \mathrm{~mL} / \mathrm{min}), \mathrm{t}_{\mathrm{R}}=13.1 \mathrm{~min}(R), \mathrm{t}_{\mathrm{R}}=$ $19.5 \min (S) ;[\alpha]^{25}{ }_{589}-116.5\left(95 \%\right.$ ee, c $\left.1.68, \mathrm{CHCl}_{3}\right)\left[{ }_{1 i t .}{ }^{3}(S\right.$ enantiomer $):[\alpha]^{27}{ }_{589}$ 
+121.24 (94.8\% ee, c 1.43, $\left.\mathrm{CHCl}_{3}\right)$ ]; IR (neat): 3079, 2913, 1689, 1642, 1462, 1270, 1197, 1179, 1141, 921, $765 \mathrm{~cm}^{-1} ;{ }^{1} \mathrm{H}$ NMR $\left(300 \mathrm{MHz}, \mathrm{CDCl}_{3}, 5.7: 1\right.$ mixture of rotamers): $\delta 2.50-3.18(\mathrm{~m}, 4 \mathrm{H}), 3.36(\mathrm{dt}, J=4.8 \mathrm{~Hz}, 11.4 \mathrm{~Hz}, 0.15 \mathrm{H}), 3.60(\mathrm{dt}, J=3.9 \mathrm{~Hz}$, $12.8 \mathrm{~Hz}, 0.85 \mathrm{H}), 3.95-4.60(\mathrm{~m}, 1 \mathrm{H}), 4.99-5.22(\mathrm{~m}, 2.30 \mathrm{H}), 5.48-6.02(\mathrm{~m}, 1.70 \mathrm{H})$, 7.03$7.42(\mathrm{~m}, 4 \mathrm{H}) ;{ }^{13} \mathrm{C}$ NMR $\left(75 \mathrm{MHz}, \mathrm{CDCl}_{3}, 5.7: 1\right.$ mixture of rotamers, signals for the minor isomer are in italic style): $\delta 156.2(\mathrm{q}, J=35.3 \mathrm{~Hz}), 135.3,135.2,133.6,133.0$, $132.8,132.5,129.2,128.8,127.5,127.1,126.6,126.3,118.4,118.2,116.7$ (q, $J=288.5$ $\mathrm{Hz}), 56.4,53.3,41.6,40.9,39.6,37.3,29.0,27.3 ; \mathrm{MS} m / z$ (relative intensity): $228\left(\mathrm{M}^{+}-\right.$ allyl, 100).

(R)-1-Allyl-6,7-dimethoxy-1,2,3,4-tetrahydroisoquinoline (3b).

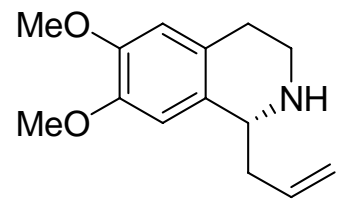

$[\alpha]_{589}^{25}+87.6$ (98\% ee, c 0.69, THF); IR (neat): 3333, 3072, 1639, 1610, 1515, 1261, 1224, 1118, 915, 857, $780 \mathrm{~cm}^{-1} ;{ }^{1} \mathrm{H}$ NMR (300 MHz, $\left.\mathrm{CDCl}_{3}\right): \delta 1.80$ (s, br, $\left.1 \mathrm{H}\right), 2.30-$ $3.15(\mathrm{~m}, 6 \mathrm{H}), 3.72(\mathrm{~s}, 6 \mathrm{H}), 3.85(\mathrm{dd}, J=2.1 \mathrm{~Hz}, 8.4 \mathrm{~Hz}, 1 \mathrm{H}), 4.98-5.16(\mathrm{~m}, 2 \mathrm{H}), 5.72(\mathrm{~m}$, 1H), $6.45(\mathrm{~s}, 1 \mathrm{H}), 6.54(\mathrm{~s}, 1 \mathrm{H}) ;{ }^{13} \mathrm{C} \mathrm{NMR}\left(75 \mathrm{MHz}, \mathrm{CDCl}_{3}\right): \delta 147.1,146.9,135.4,130.3$, $127.2,117.6,111.5,108.8,55.7,55.5,54.5,40.8,40.5,29.2 ; \mathrm{MS} m / z$ (relative intensity): $233\left(\mathrm{M}^{+}, 0.5\right), 232,\left(\mathrm{M}^{+}-\mathrm{H}, 2.5\right), 192\left(\mathrm{M}^{+}\right.$-allyl, 100); Enantiomeric excess was determined by HPLC analysis of its trifluoroacetamide: (hexane $/ i-\operatorname{PrOH}=98 / 2$, flow rate $=1 \mathrm{~mL} / \mathrm{min}), \mathrm{t}_{\mathrm{R}}=17.6 \min (S), \mathrm{t}_{\mathrm{R}}=23.6 \min (R) ;[\alpha]_{589}^{25}-113.8\left(98 \% e e, \mathrm{c} 0.65, \mathrm{CHCl}_{3}\right)$ [lit. ${ }^{3}$ ( $S$ enantiomer): $[\alpha]^{25}{ }_{589}+106.8\left(95 \% e e\right.$, c $\left.\left.1.29, \mathrm{CHCl}_{3}\right)\right]$; IR (neat): 3075,2937 , $2837,1688,1642,1520,1464,1256,1232,1178,1195,1178,1140,1118,915,857,778$, 
754, 668, $654 \mathrm{~cm}^{-1} ;{ }^{1} \mathrm{H}$ NMR (300 MHz, $\mathrm{CDCl}_{3}, 6.1: 1$ mixture of rotamers): $\delta 2.48-2.78$ (m, 3H), 2.86-3.02 (m, 1H), 3.29 (dt, $J=4.9 \mathrm{~Hz}, 12.6 \mathrm{~Hz}, 0.14 \mathrm{H}), 3.56$ (ddd, $J=4.2 \mathrm{~Hz}$, $12.2 \mathrm{~Hz}, 14.1 \mathrm{~Hz}, 0.86 \mathrm{H}), 3.82(\mathrm{~s}, 3 \mathrm{H}), 3.83(\mathrm{~s}, 3 \mathrm{H}), 3.95-4.05$ (m, 0.86H), $4.53(\mathrm{~m}$, $0.14 \mathrm{H}), 4.88(\mathrm{t}, J=7.1 \mathrm{~Hz}, 0.14 \mathrm{H}), 4.98-5.13(\mathrm{~m}, 2 \mathrm{H}), 5.51(\mathrm{dd}, J=5.2 \mathrm{~Hz}, 9.2 \mathrm{~Hz}$, $0.86 \mathrm{H}), 5.69-5.88(\mathrm{~m}, 1 \mathrm{H}), 6.54(\mathrm{~s}, 0.14 \mathrm{H}), 6.56(\mathrm{~s}, 0.86 \mathrm{H}), 6.59(\mathrm{~s}, 0.14 \mathrm{H}), 6.60(\mathrm{~s}$, $0.86 \mathrm{H}) ;{ }^{13} \mathrm{C}$ NMR $\left(75 \mathrm{MHz}, \mathrm{CDCl}_{3}, 6.1: 1\right.$ mixture of rotamers, signals for the minor isomer are in italic style): $\delta 155.9(\mathrm{q}, J=35.6 \mathrm{~Hz}), 148.4,148.1,147.8,147.5,133.7$, $133.2,127.2,127.1,124.9,124.5,118.8,118.2,116.5(\mathrm{q}, J=287.9 \mathrm{~Hz}), 111.5,111.1$, $109.7,109.5,56.0,55.9,53.0,41.7,40.9,39.6,37.2,28.7,27.0 ;$ MS $m / z$ (relative intensity): 228 ( $\mathrm{M}^{+}$-allyl, 100).

\section{(R)-5-Allyl-5,6,7,8-tetrahydro-[1,3]dioxolo[4,5-g]isoquinoline (3c).}

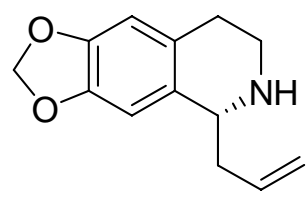

$[\alpha]_{589}^{25}+97.8$ (98\% ee, c 0.91, THF); IR (neat): 3305, 3073, 2910, 1638, 1503, 1484, $1248,1229,1040,936,861,805 \mathrm{~cm}^{-1} ;{ }^{1} \mathrm{H}$ NMR $\left(300 \mathrm{MHz}, \mathrm{CDCl}_{3}\right): \delta 1.74(\mathrm{~s}, \mathrm{br}, 1 \mathrm{H})$, 2.31-3.22 (m, 6H), 3.89 (dd, $J=2.7 \mathrm{~Hz}, 8.4 \mathrm{~Hz}, 1 \mathrm{H}), 5.07-5.15(\mathrm{~m}, 2 \mathrm{H}), 5.79(\mathrm{~m}, 1 \mathrm{H})$, $5.84(\mathrm{~s}, 2 \mathrm{H}), 6.50(\mathrm{~s}, 1 \mathrm{H}), 6.60(\mathrm{~s}, 1 \mathrm{H}) ;{ }^{13} \mathrm{C} \mathrm{NMR}\left(75 \mathrm{MHz}, \mathrm{CDCl}_{3}\right): \delta 145.6,145.5$, 135.4, 131.6. 128.4, 117.8, 108.7, 105.9, 100.5, 55.0, 40.9, 40.6, 30.0; MS $m / z$ (relative intensity): $217\left(\mathrm{M}^{+}, 0.3\right), 216,\left(\mathrm{M}^{+}-\mathrm{H}, 1.6\right), 176\left(\mathrm{M}^{+}\right.$-allyl, 100); HRMS $m / z$ Calcd. for $\mathrm{C}_{13} \mathrm{H}_{14} \mathrm{NO}_{2}\left(\mathrm{M}^{+}-\mathrm{H}\right): 216.1025$. Found: 216.1027; Enantiomeric excess was determined by HPLC analysis of its trifluoroacetamide: (hexane $/ i-\mathrm{PrOH}=98 / 2$, flow rate $=1 \mathrm{~mL} / \mathrm{min}$ ), $\mathrm{t}_{\mathrm{R}}=19.8 \min (S), \mathrm{t}_{\mathrm{R}}=22.1 \min (R) ;[\alpha]^{25}{ }_{589}-94.1\left(98 \%\right.$ ee, c 1.31, $\left.\mathrm{CHCl}_{3}\right) ; \mathrm{IR}$ (neat): 
$2910,1690,1642,1506,1487,1240,1199,1140,1040,923,864,753,668,650 \mathrm{~cm}^{-1} ;{ }^{1} \mathrm{H}$ NMR (300 MHz, $\mathrm{CDCl}_{3}, 6.3: 1$ mixture of rotamers): $\delta 2.48-3.04(\mathrm{~m}, 4 \mathrm{H}), 3.32(\mathrm{dt}, J=$ 4.6 Hz, 11.6 Hz, 0.14H), 3.55 (dt, $J=3.5 \mathrm{~Hz}, 11.3 \mathrm{~Hz}, 0.86 \mathrm{H}), 3.91-4.52(\mathrm{~m}, 1 \mathrm{H}), 4.82-$ $5.54(\mathrm{~m}, 3 \mathrm{H}), 5.70-5.86(\mathrm{~m}, 1 \mathrm{H}), 5.89(\mathrm{~s}, 2 \mathrm{H}), 6.53-6.58(\mathrm{~m}, 2 \mathrm{H}) ;{ }^{13} \mathrm{C} \mathrm{NMR}(75 \mathrm{MHz}$, $\mathrm{CDCl}_{3}, 6.3: 1$ mixture of rotamers, signals for the minor isomer are in italic style): $\delta 156.0$ $(\mathrm{q}, J=30.0 \mathrm{~Hz}), 149.0,146.8,146.6,144.4,133.6,133.1,128.4,128.3,126.4,125.9$, 119.0, 118.4, $116.6(\mathrm{q}, J=285 \mathrm{~Hz}), 108.9,108.4,106.9,106.7,101.1,56.0,53.4,41.7$, 41.0, 39.7, 37.3, 29.2, 27.4; MS $m / z$ (relative intensity): $313\left(\mathrm{M}^{+}, 1.5\right), 272\left(\mathrm{M}^{+}\right.$-allyl, 100); HRMS $m / z$ Calcd. for $\mathrm{C}_{15} \mathrm{H}_{14} \mathrm{~F}_{3} \mathrm{NO}_{3}\left(\mathrm{M}^{+}-\mathrm{H}\right): 313.0926$. Found: 313.0934 .

(R)-1-Allyl-6,7-dichloro-1,2,3,4-tetrahydroisoquinoline (3d).

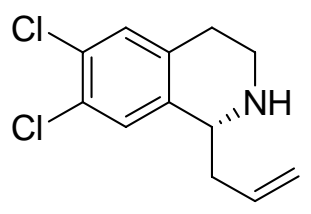

$[\alpha]_{589}^{25}+117.6$ (95\% ee, c 0.91, THF); IR (neat): 3339, 3076, 2926, 1640, 1472, 1136, 917, $667 \mathrm{~cm}^{-1} ;{ }^{1} \mathrm{H}$ NMR (300 MHz, $\left.\mathrm{CDCl}_{3}\right): \delta 1.75$ (s, br, $\left.1 \mathrm{H}\right), 2.31-3.33(\mathrm{~m}, 6 \mathrm{H}), 3.90$ (dd, $J=3.0 \mathrm{~Hz}, 8.7 \mathrm{~Hz}, 1 \mathrm{H}), 5.08-5.15(\mathrm{~m}, 1 \mathrm{H}), 5.74(\mathrm{~m}, 1 \mathrm{H}), 7.11(\mathrm{~s}, 1 \mathrm{H}), 7.19(\mathrm{~s}, 1 \mathrm{H})$; ${ }^{13} \mathrm{C}$ NMR $\left(75 \mathrm{MHz}, \mathrm{CDCl}_{3}\right): \delta 138.9,135.8,134.8,130.9,129.7,129.5,127.9,118.6$, 54.6, 40.7, 40.4, 29.3; MS m/z (relative intensity): $241\left(\mathrm{M}^{+}, 0.3\right), 240,\left(\mathrm{M}^{+}-\mathrm{H}, 1.1\right), 200$ ( $\mathrm{M}^{+}$-allyl, 100); HRMS $m / z$ Calcd. for $\mathrm{C}_{12} \mathrm{H}_{12} \mathrm{NCl}_{2}\left(\mathrm{M}^{+}-\mathrm{H}\right)$ : 240.0347. Found: 240.0345; Enantiomeric excess was determined by ${ }^{19} \mathrm{~F}$ NMR of its MTPA-amide $[\delta$-69.55 $(R)$, $70.75(\mathrm{~S})]$ 
(R)-1-Allyl-7-nitro-1,2,3,4-tetrahydroisoquinoline (3e).

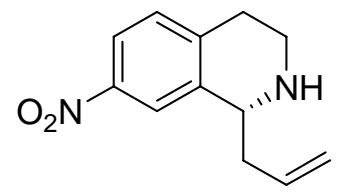

$[\alpha]_{589}^{25}+144\left(99 \%\right.$ ee, c 3.4, $\left.\mathrm{CHCl}_{3}\right)$; IR (neat): 3344, 3074, 2927, 1640, 1587, 1518, 1345, 1279, 1134, 903, $739 \mathrm{~cm}^{-1} ;{ }^{1} \mathrm{H}$ NMR (300 MHz, $\left.\mathrm{CDCl}_{3}\right): \delta 2.03$ (s, br, 1H), 2.44$3.32(\mathrm{~m}, 6 \mathrm{H}), 4.08(\mathrm{dd}, J=3.1 \mathrm{~Hz}, 8.6 \mathrm{~Hz}, 1 \mathrm{H}), 5.14-5.24(\mathrm{~m}, 2 \mathrm{H}), 5.81(\mathrm{~m}, 1 \mathrm{H}), 7.23(\mathrm{~d}$, $J=8.0 \mathrm{~Hz}, 1 \mathrm{H}), 7.97(\mathrm{dd}, J=2.1 \mathrm{~Hz}, 8.0 \mathrm{~Hz}, 1 \mathrm{H}) ; 8.05(\mathrm{~d}, J=2.1 \mathrm{~Hz}, 1 \mathrm{H}) ;{ }^{13} \mathrm{C} \mathrm{NMR}$ $\left(75 \mathrm{MHz}, \mathrm{CDCl}_{3}\right): \delta 146.2,143.4,140.0,134.4,130.2,121.3,121.0,118.9,55.0,40.4$, 40.2, 30.1; MS m/z (relative intensity): $218\left(\mathrm{M}^{+}, 0.1\right), 217,\left(\mathrm{M}^{+}-\mathrm{H}, 0.6\right), 177\left(\mathrm{M}^{+}\right.$-allyl, 100), $131\left(\mathrm{M}^{+}-\mathrm{NO}_{2}\right.$-allyl, 37); HRMS $m / z$ Calcd. for $\mathrm{C}_{9} \mathrm{H}_{9} \mathrm{~N}_{2} \mathrm{O}_{2}\left(\mathrm{M}^{+}\right.$-allyl): 177.0664 . Found: 177.0662; Enantiomeric excess was determined by HPLC analysis of its trifluoroacetamide: $($ hexane $/ i-\mathrm{PrOH}=98 / 2$, flow rate $=1 \mathrm{~mL} / \mathrm{min}), \mathrm{t}_{\mathrm{R}}=28.0 \mathrm{~min}(S), \mathrm{t}_{\mathrm{R}}=$ $29.8 \min (R) .[\alpha]^{25}{ }_{589}-137\left(99 \%\right.$ ee, c 1.67, $\left.\mathrm{CHCl}_{3}\right)$; IR (neat): 2959, 1692, 1640, 1525 , $1467,1346,1282,1260,1195,1158,1141,1045,1000,944,928,895,834,738 \mathrm{~cm}^{-1} ;{ }^{1} \mathrm{H}$ NMR (300 MHz, $\mathrm{CDCl}_{3}, 5.6: 1$ mixture of rotamers): $\delta$ 2.52-2.79 (m, 2H), 2.89-3.16 (m, 2H), 3.35 (ddd, $J=5.4 \mathrm{~Hz}, 11.1 \mathrm{~Hz}, 13.5 \mathrm{~Hz}, 0.18 \mathrm{H}), 3.60$ (ddd, $J=4.8 \mathrm{~Hz}, 11.1 \mathrm{~Hz}$, 14.4 Hz, 0.82H), 4.02-4.65 (m, 1H), 4.99-5.85 (m, 4H), 7.25-7.36 (m, 1H), 7.95-8.07 (m, $2 \mathrm{H}) ;{ }^{13} \mathrm{C} \mathrm{NMR}\left(75 \mathrm{MHz}, \mathrm{CDCl}_{3}, 5.6: 1\right.$ mixture of rotamers, signals for the minor isomer are in italic style): $\delta 156.0(\mathrm{q}, J=36.0 \mathrm{~Hz}), 146.6,146.3,140.7,140.3,136.8,136.7$, $132.6,132.0,130.5,130.0,122.4,122.3,122.0,121.9,119.9,119.1,116.3(\mathrm{q}, J=288$ $\mathrm{Hz}$ ), 56.0, 53.0, 41.3, 40.6, 38.9, 36.3, 29.3, 27.5; MS $m / z$ (relative intensity): $314\left(\mathrm{M}^{+}\right.$, 0.1), $273\left(\mathrm{M}^{+}\right.$-allyl, 100); HRMS m/z Calcd. for $\mathrm{C}_{11} \mathrm{H}_{8} \mathrm{~F}_{3} \mathrm{~N}_{2} \mathrm{O}_{3}\left(\mathrm{M}^{+}\right.$-allyl): 273.0487 . Found: 273.0496 . 
(R)-1-Allyl-2,3,4,9-tetrahydro-1H-pyrido[3,4-b]indole (3f).

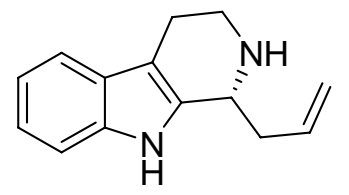

${ }^{1} \mathrm{H}$ NMR (300 MHz, $\left.\mathrm{CDCl}_{3}\right): \delta 2.25(\mathrm{~s}, \mathrm{br}, 1 \mathrm{H}), 2.44-3.43(\mathrm{~m}, 6 \mathrm{H}), 4.14(\mathrm{t}, \mathrm{J}=6.3 \mathrm{~Hz}$, 1H), 5.16-5.30 (m, 2H), $5.87(\mathrm{~m}, 1 \mathrm{H}), 7.08-7.22(\mathrm{~m}, 2 \mathrm{H}), 7.27(\mathrm{~d}, \mathrm{~J}=7.2 \mathrm{~Hz}, 1 \mathrm{H}), 7.51$ $(\mathrm{d}, \mathrm{J}=7.2 \mathrm{~Hz}, 1 \mathrm{H}), 8.40(\mathrm{~s}, 1 \mathrm{H}) ;{ }^{13} \mathrm{C} \mathrm{NMR}\left(75 \mathrm{MHz}, \mathrm{CDCl}_{3}\right): \delta 135.7,135.6,134.9$, $127.3,121.5,119.2,118.5,118.1,116.9,110.8,109.1,51.9,42.6,39.3,22.6$; $3 f$ was derivatized by sequential treatment with $\left(\mathrm{CF}_{3} \mathrm{CO}\right)_{2} \mathrm{O} /$ pyridine and $\mathrm{TsCl} / \mathrm{NaH}$. Enantiomeric excess was then determined by chiral HPLC analysis of that derivative: (hexane $/ i-\mathrm{PrOH}=98 / 2$, flow rate $\left.=1 \mathrm{~mL} / \mathrm{min}), \mathrm{t}_{\mathrm{R}}=8.7 \mathrm{~min}(S), \mathrm{t}_{\mathrm{R}}=19.7 \mathrm{~min}(R).\right)$; $[\alpha]_{589}^{25}-173.2\left(94 \%\right.$ ee, c 0.50, $\left.\mathrm{CHCl}_{3}\right)$; IR (neat): 3074, 2959, 2925, 1692, 1643, 1598, $1452,1377,1208,1174,1143,1089,1041,1022,800,756,666,584,572,543 \mathrm{~cm}^{-1} ;{ }^{1} \mathrm{H}$ NMR (300 MHz, $\mathrm{CDCl}_{3}, 5: 1$ mixture of rotamers): $\delta 2.27(\mathrm{~s}, 2.5 \mathrm{H}), 2.28(\mathrm{~s}, 0.5 \mathrm{H}), 2.42-$ $2.91(\mathrm{~m}, 3 \mathrm{H}), 3.11-3.66(\mathrm{~m}, 2 \mathrm{H}), 4.08-4.80(\mathrm{~m}, 1 \mathrm{H}), 5.04-5.16(\mathrm{~m}, 2 \mathrm{H})$, 5.68-5.98 (m, 1.17H), $6.39(\mathrm{dd}, J=2.8 \mathrm{~Hz}, 10.6 \mathrm{~Hz}, 0.83 \mathrm{H}), 7.14(\mathrm{~d}, J=8.3 \mathrm{~Hz}, 2 \mathrm{H}), 7.19-7.36(\mathrm{~m}$, $3 \mathrm{H}), 7.55(\mathrm{~d}, J=8.3 \mathrm{~Hz}, 0.34 \mathrm{H}), 7.67(\mathrm{~d}, J=8.3 \mathrm{~Hz}, 1.66 \mathrm{H}), 8.09(\mathrm{~d}, J=8.1 \mathrm{~Hz}, 0.17 \mathrm{H})$, $8.17(\mathrm{~d}, \mathrm{~J}=8.3 \mathrm{~Hz}, 0.83 \mathrm{H}) ;{ }^{13} \mathrm{C} \mathrm{NMR}\left(75 \mathrm{MHz}, \mathrm{CDCl}_{3}, 5: 1\right.$ mixture of rotamers, signals for the minor isomer are in italic style): $\delta 156.6(\mathrm{q}, J=36.0 \mathrm{~Hz}), 145.3,145.2,136.9$, $136.7,134.7,134.3,134.0,133.5,133.0,130.0,129.9,129.5,129.4,126.8,126.5,125.5$, $125.3,124.3,119.0,118.8,118.6,118.2,117.2,115.6,115.4,116.5$ (q, $J=269 \mathrm{~Hz}), 54.2$, 51.0, 39.9, 39.2, 38.2, 36.0, 22.7, 22.2, 21.6, 20.4; MS m/z (relative intensity): $462\left(\mathrm{M}^{+}\right.$, 1.2), $421\left(\mathrm{M}^{+}\right.$-allyl, 100); HRMS m/z Calcd. for $\mathrm{C}_{20} \mathrm{H}_{16} \mathrm{~F}_{3} \mathrm{~N}_{2} \mathrm{O}_{3} \mathrm{~S}$ ( $\mathrm{M}^{+}$-allyl): 421.0834 . Found: 421.0831 . 
(R)-1-Allyl-9-tosyl-2,3,4,9-tetrahydro-1H-pyrido[3,4-b]indole (3g).

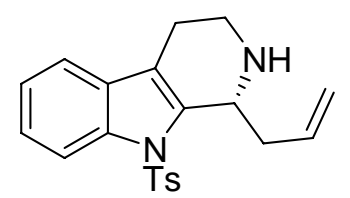

$[\alpha]_{589}^{25}-202.4\left(94 \%\right.$ ee, c 1.8, $\left.\mathrm{CHCl}_{3}\right)$; IR (neat): 3337, 3070, 2925, 1639, 1597, 1450, 1366, 1172, 754, 667, 584, $573 \mathrm{~cm}^{-1} ;{ }^{1} \mathrm{H}$ NMR (300 MHz, $\left.\mathrm{CDCl}_{3}\right): \delta 2.06$ (s, br, 1H), $2.26(\mathrm{~s}, 3 \mathrm{H}), 2.41-3.15(\mathrm{~m}, 6 \mathrm{H}), 4.53(\mathrm{~d}, J=9.3 \mathrm{~Hz}, 1 \mathrm{H}), 5.14-5.20(\mathrm{~m}, 2 \mathrm{H}), 5.94(\mathrm{~m}$, 1H), 7.09 (d, $J=8.1 \mathrm{~Hz}, 2 \mathrm{H}), 7.19-7.34(\mathrm{~m}, 3 \mathrm{H}), 7.51$ (d, $J=8.1 \mathrm{~Hz}, 2 \mathrm{H}), 8.11(\mathrm{~d}, J=$ $6.1 \mathrm{~Hz}, 1 \mathrm{H}) ;{ }^{13} \mathrm{C} \mathrm{NMR}\left(75 \mathrm{MHz}, \mathrm{CDCl}_{3}\right): \delta 144.6,137.8,136.8,136.0,134.9,130.7$, $129.6,126.4,124.5,123.8,119.3,118.3,117.7,115.5,52.6,39.0,37.2,22.5,21.5$; $\mathrm{MS}(\mathrm{CI}) \mathrm{m} / \mathrm{e}$ (relative intensity): $367\left([\mathrm{M}+1]^{+}, 100\right), 325\left(\mathrm{M}^{+}\right.$-allyl, 14), $192\left(\mathrm{M}^{+}\right.$-allyl, 100); HRMS $m / z$ Calcd for $\mathrm{C}_{18} \mathrm{H}_{17} \mathrm{~N}_{2} \mathrm{O}_{2} \mathrm{~S}$ ( $\mathrm{M}^{+}$-allyl): 325.1011. Found: 325.1012; Enantiomeric excess was determined by HPLC analysis of its trifluoroacetamide: (hexane $/ i-\mathrm{PrOH}=98 / 2$, flow rate $=1 \mathrm{~mL} / \mathrm{min}), \mathrm{t}_{\mathrm{R}}=8.7 \mathrm{~min}(S), \mathrm{t}_{\mathrm{R}}=19.7 \mathrm{~min}(R)$;

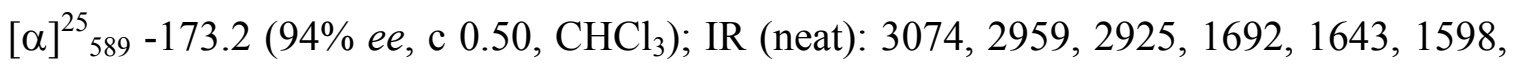
$1452,1377,1208,1174,1143,1089,1041,1022,800,756,666,584,572,543 \mathrm{~cm}^{-1} ;{ }^{1} \mathrm{H}$ NMR (300 MHz, $\mathrm{CDCl}_{3}, 5: 1$ mixture of rotamers): $\delta 2.27(\mathrm{~s}, 2.5 \mathrm{H}), 2.28(\mathrm{~s}, 0.5 \mathrm{H}), 2.42-$ $2.91(\mathrm{~m}, 3 \mathrm{H}), 3.11-3.66(\mathrm{~m}, 2 \mathrm{H}), 4.08-4.80(\mathrm{~m}, 1 \mathrm{H}), 5.04-5.16(\mathrm{~m}, 2 \mathrm{H})$, 5.68-5.98 (m, 1.17H), 6.39 (dd, $J=2.8 \mathrm{~Hz}, 10.6 \mathrm{~Hz}, 0.83 \mathrm{H}), 7.14$ (d, $J=8.3 \mathrm{~Hz}, 2 \mathrm{H}), 7.19-7.36$ (m, $3 \mathrm{H}), 7.55(\mathrm{~d}, J=8.3 \mathrm{~Hz}, 0.34 \mathrm{H}), 7.67(\mathrm{~d}, J=8.3 \mathrm{~Hz}, 1.66 \mathrm{H}), 8.09(\mathrm{~d}, J=8.1 \mathrm{~Hz}, 0.17 \mathrm{H})$, $8.17(\mathrm{~d}, \mathrm{~J}=8.3 \mathrm{~Hz}, 0.83 \mathrm{H}) ;{ }^{13} \mathrm{C} \mathrm{NMR}\left(75 \mathrm{MHz}, \mathrm{CDCl}_{3}, 5: 1\right.$ mixture of rotamers, signals for the minor isomer are in italic style): $\delta 156.6(\mathrm{q}, J=36.0 \mathrm{~Hz}), 145.3,145.2,136.9$, $136.7,134.7,134.3,134.0,133.5,133.0,130.0,129.9,129.5,129.4,126.8,126.5,125.5$, 
125.3, 124.3, 119.0, 118.8,118.6, 118.2, 117.2, 115.6, 115.4, 116.5 (q, $J=269 \mathrm{~Hz}), 54.2$, 51.0, 39.9, 39.2, 38.2, 36.0, 22.7, 22.2, 21.6, 20.4; MS m/z (relative intensity): $462\left(\mathrm{M}^{+}\right.$, 1.2), $421\left(\mathrm{M}^{+}\right.$-allyl, 100); HRMS $m / z$ Calcd. for $\mathrm{C}_{20} \mathrm{H}_{16} \mathrm{~F}_{3} \mathrm{~N}_{2} \mathrm{O}_{3} \mathrm{~S}$ ( $\mathrm{M}^{+}$-allyl): 421.0834 . Found: 421.0831.

\section{(S)-2-allyl-1-tosylpiperidine (3h).}

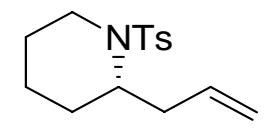

Allylation of imine $\mathbf{1 h}(0.24 \mathrm{mmol})$ was accomplished according to the general procedure. To the crude reaction mixture was added TsCl (381 mg, $2 \mathrm{mmol}$ ) and DMAP (244 mg, 2 mmol). The reaction was stirred at room temperature overnight. Solvent was removed under reduced pressure. The residue was purified by column chromatography (1:1 hexane/EtOAc) to give $43 \mathrm{mg}$ of $3 \mathbf{i}(65 \%)$. [ $\alpha]^{25}{ }_{589}-42.2\left(91 \%\right.$ ee, c $\left.2.22, \mathrm{CH}_{2} \mathrm{Cl}_{2}\right)\left[\mathrm{lit}^{3}{ }^{3}\right.$ ( $R$ enantiomer): $[\alpha]^{27}{ }_{589}+35.1$ (89.4\% ee, c 0.40, $\left.\left.\mathrm{CH}_{2} \mathrm{Cl}_{2}\right)\right]$; IR (neat): 3066, 2941, 2865 , $1628,1600,1452,1380,1326,1150,926,814,658,550 \mathrm{~cm}^{-1} ;{ }^{1} \mathrm{H}$ NMR $(300 \mathrm{MHz}$, $\left.\mathrm{CDCl}_{3}\right): \delta 1.16-1.60(\mathrm{~m}, 6 \mathrm{H}), 2.26(\mathrm{t}, J=7.7 \mathrm{~Hz}, 2 \mathrm{H}), 2.38(\mathrm{~s}, 3 \mathrm{H}), 2.94(\mathrm{dt}, J=2.6 \mathrm{~Hz}$, 13.7 Hz, 1H), 3.73 (dd, $J=3.7 \mathrm{~Hz}, 13.7 \mathrm{~Hz}, 1 \mathrm{H}), 4.07$ (m, 1H), 4.97-5.02 (m, 2H), 5.65 $(\mathrm{m}, 1 \mathrm{H}), 7.24(\mathrm{~d}, J=8.0 \mathrm{~Hz}, 2 \mathrm{H}), 7.68(\mathrm{~d}, J=8.0 \mathrm{~Hz}, 2 \mathrm{H}) ;{ }^{13} \mathrm{C} \mathrm{NMR}\left(75 \mathrm{MHz}, \mathrm{CDCl}_{3}\right)$ : $\delta 142.7,138.7,134.9,129.5,126.9,117.0,52.4,40.6,33.9,26.5,24.5,21.4,18.1 ; \mathrm{MS}$ (CI) $m / z$ (relative intensity): $280\left([\mathrm{M}+1]^{+}, 100\right), 238,\left(\mathrm{M}^{+}\right.$-allyl, 37); Enantiomeric excess was determined by HPLC analysis: (hexane $/ i-\mathrm{PrOH}=99.5 / 0.5$, flow rate $=1 \mathrm{~mL} / \mathrm{min}$ ), $\mathrm{t}_{\mathrm{R}}$ $=47.5 \min (R), \mathrm{t}_{\mathrm{R}}=51.2 \min (S)$. 


\section{(S)-tert-butyl 2-allylpyrrolidine-1-carboxylate (3i)}

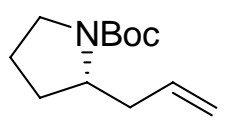

Allylation of imine $1 \mathbf{i}(0.20 \mathrm{mmol})$ was accomplished according to the general procedure. To the crude reaction mixture was added (Boc) $)_{2} \mathrm{O}(436 \mathrm{mg}, 2 \mathrm{mmol})$ and $\mathrm{NEt}_{3}(0.2 \mathrm{~mL})$. The reaction was stirred at room temperature overnight. Solvent was removed under reduced pressure. The residue was purified by column chromatography $(3: 1$ hexane/EtOAc) to give $30 \mathrm{mg}$ of $3 \mathbf{i}(71 \%)$. $[\alpha]^{25}{ }_{589}-45.4\left(92 \%\right.$ ee, c $\left.1.26, \mathrm{CHCl}_{3}\right)\left[\right.$ lit. $^{4}$ $[\alpha]_{589}^{25}-32.4$ (c 1.56, $\left.\left.\mathrm{CHCl}_{3}\right)\right]$; IR (neat): 3077, 2976, 1694, 1641, 1394, 1366, 1173, $1114 \mathrm{~cm}^{-1} ;{ }^{1} \mathrm{H}$ NMR $\left(300 \mathrm{MHz}, \mathrm{CDCl}_{3}\right): \delta 1.43(\mathrm{~s}, 9 \mathrm{H}), 1.54-1.84(\mathrm{~m}, 4 \mathrm{H}), 2.00-2.55(\mathrm{~m}$, 2H), 3.21-3.42 (m, 2H), 3.66-3.85 (m, 1H), 4.98-5.05 (m, 2H), $5.72(\mathrm{~m}, 1 \mathrm{H}) ;{ }^{13} \mathrm{C} \mathrm{NMR}$ $\left(75 \mathrm{MHz}, \mathrm{CDCl}_{3}\right): \delta 23.1,28.5,30.0,38.1,45.6,53.7,78.8,116.9,135.2,145.0 ; \mathrm{MS} m / z$ (relative intensity): $211\left(\mathrm{M}^{+}, 0.1\right), 170\left(\mathrm{M}^{+}\right.$-allyl, 42), 114 (100); Enantiomeric excess was determined by HPLC analysis: (hexane $/ i-\mathrm{PrOH}=99.5 / 0.5$, flow rate $=0.3 \mathrm{~mL} / \mathrm{min}$ ), $\mathrm{t}_{\mathrm{R}}=30.0 \min (R), \mathrm{t}_{\mathrm{R}}=32.3 \min (S)$.

\section{(R)-3-(6,7-dimethoxy-1,2,3,4-tetrahydroisoquinolin-1-yl)propan-1-ol}

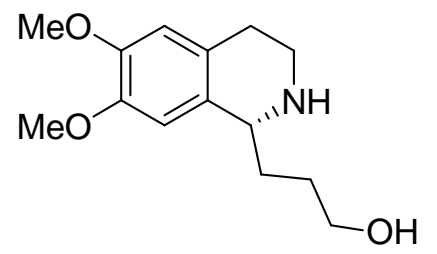

To a solution of amine $3 \mathbf{b}(110 \mathrm{mg}, 0.47 \mathrm{mmol})$ in THF $(2 \mathrm{~mL})$ was added 9-BBN (3 mL, $0.5 \mathrm{M}$ in THF) dropwise. The reaction was stirred at room temperature for 3 hours. A mixture of aqueous $\mathrm{NaOH}(3 \mathrm{M}, 5 \mathrm{~mL})$ and $30 \% \mathrm{H}_{2} \mathrm{O}_{2}(5 \mathrm{~mL})$ were added at room temperature. The exothermic reaction caused the temperature to quickly rise to $55^{\circ} \mathrm{C}$ and 
this temperature was maintained for 25 minutes with external heat. The reaction mixture was then cooled to room temperature and extracted with $\mathrm{Et}_{2} \mathrm{O}(3 \times 5 \mathrm{~mL})$. The combined ethereal extracts were washed with brine $(10 \mathrm{~mL})$ and dried over $\mathrm{Na}_{2} \mathrm{SO}_{4}$. Purification by flash column chromatography $\left(10: 1 \mathrm{CH}_{2} \mathrm{Cl}_{2} / \mathrm{MeOH}, 0.5 \% \mathrm{NH}_{4} \mathrm{OH}\right)$ gave the intermediate alcohol (96 mg, $81 \%$ ). IR (neat): 3303, 2934, 1611, 1515, 1257, 1223, $1115 \mathrm{~cm}^{-1} ;{ }^{1} \mathrm{H}$ NMR (300 MHz, $\left.\mathrm{CDCl}_{3}\right): \delta 1.62-2.00(\mathrm{~m}, 4 \mathrm{H}), 2.56-2.81(\mathrm{~m}, 2 \mathrm{H}), 2.95-3.22(\mathrm{~m}, 2 \mathrm{H})$, 3.46-3.66 (m, 2H), $3.81(\mathrm{~s}, 6 \mathrm{H}), 3.91(\mathrm{dd}, J=4.5 \mathrm{~Hz}, 7.2 \mathrm{~Hz}, 1 \mathrm{H}), 4.72(\mathrm{~s}, \mathrm{br}, 2 \mathrm{H}), 6.52$ $(\mathrm{s}, 1 \mathrm{H}), 6.55(\mathrm{~s}, 1 \mathrm{H}) ;{ }^{13} \mathrm{C} \mathrm{NMR}\left(75 \mathrm{MHz}, \mathrm{CDCl}_{3}\right): \delta 147.5,147.4,130.2,126.6,111.6$, 109.3, 62.8, 56.0, 55.8, 55.4, 39.7, 35.5, 30.5, 28.6; MS $m / z$ (relative intensity): $251\left(\mathrm{M}^{+}\right.$, 0.5), $250\left(\mathrm{M}^{+}-\mathrm{H}, 2.4\right), 232\left(\mathrm{M}^{+}-\mathrm{H}-\mathrm{H}_{2} \mathrm{O}, 6.5\right), 192\left(\mathrm{M}^{+}-\mathrm{CH}_{2} \mathrm{CH}_{2} \mathrm{CH}_{2} \mathrm{OH}, 100\right)$. HRMS m/z Calcd. for $\mathrm{C}_{14} \mathrm{H}_{20} \mathrm{NO}_{3}\left(\mathrm{M}^{+}-\mathrm{H}\right): 250.1443$. Found: 250.1444 .

\section{(+)-Crispine A (4).}

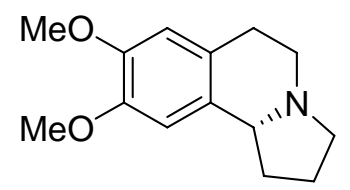

The alcohol was stirred with $\mathrm{PPh}_{3}(262 \mathrm{mg}, 1.0 \mathrm{mmol}), \mathrm{CBr}_{4}(331 \mathrm{mg}, 1.0 \mathrm{mmol})$ and $i$ $\operatorname{Pr}_{2}$ NEt $(0.3 \mathrm{~mL})$ in THF $(10 \mathrm{~mL})$ at room temperature for 2 hours, followed by removal of the solvent under reduced pressure. Flash column chromatography $(3: 1 \mathrm{EtOAc} / \mathrm{MeOH}$, $1 \% \mathrm{NH}_{4} \mathrm{OH}$ ) yielded the title compound (73 mg, $66 \%$ yield for 2 steps). $[\alpha]^{25}{ }_{589}+96.9$ (c 1.1, $\left.\mathrm{CHCl}_{3}\right)\left[\right.$ lit. $^{5}[\alpha]^{23}{ }_{589}+100.4\left(>99 \%\right.$ ee, c 1, $\left.\left.\mathrm{CHCl}_{3}\right)\right]$; IR(neat): 3303, 2934, 1611, 1515, 1257, 1223, $1115 \mathrm{~cm}^{-1} ;{ }^{1} \mathrm{H}$ NMR (300 MHz, $\left.\mathrm{CDCl}_{3}\right): \delta 1.62-2.00(\mathrm{~m}, 3 \mathrm{H}), 2.23-$ $2.37(\mathrm{~m}, 1 \mathrm{H}), 2.48-2.77(\mathrm{~m}, 3 \mathrm{H}), 2.92-3.21(\mathrm{~m}, 3 \mathrm{H}), 3.41(\mathrm{t}, J=8.4 \mathrm{~Hz}, 1 \mathrm{H}), 3.82(\mathrm{~s}, 6 \mathrm{H})$,

$6.54(\mathrm{~s}, 1 \mathrm{H}), 6.58(\mathrm{~s}, 1 \mathrm{H}) ;{ }^{13} \mathrm{C} \mathrm{NMR}\left(75 \mathrm{MHz}, \mathrm{CDCl}_{3}\right): \delta 147.2,147.1,130.9,126.2$, 
$111.2,108.8,62.9,55.9,55.8,53.0,48.2,30.4,27.9,22.2 ; \mathrm{MS} \mathrm{m} / \mathrm{z}$ (relative intensity):

$233\left(\mathrm{M}^{+}, 43\right), 232\left(\mathrm{M}^{+}-\mathrm{H}, 100\right), 205\left(\mathrm{M}^{+}-\mathrm{C}_{2} \mathrm{H}_{4}, 43\right), 190\left(\mathrm{M}^{+}-\mathrm{CH}_{2} \mathrm{CH}_{2} \mathrm{CH}_{2} \mathrm{OH}, 33\right)$.

\section{R-(-)-Coniine'HCl (5).}

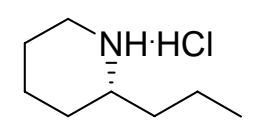

Allylation of imine $\mathbf{1 h}(0.32 \mathrm{mmol})$ was accomplished according to the general procedure. To the crude reaction mixture was added $10 \% \mathrm{Pd} / \mathrm{C}(5 \mathrm{~mol} \%)$ and $\mathrm{MeOH}(3 \mathrm{~mL})$. The suspension was stirred at room temperature under $\mathrm{H}_{2}$ atmosphere for 12 hours before the addition of $\mathrm{HCl} / \mathrm{Et}_{2} \mathrm{O}(4 \mathrm{~mL}, 0.5 \mathrm{M})$. (R)-(-)-Coniine $\mathrm{HCl}(5)$ was obtained after column chromatography on silica gel $\left(10: 1 \sim 6: 1 \mathrm{CH}_{2} \mathrm{Cl}_{2} / \mathrm{MeOH}\right)$. $[\alpha]^{25}{ }_{589}-6.8$ (c 0.34 , EtOH) [lit. ${ }^{6}[\alpha]^{22}{ }_{589}-7.1$ (c 1.0, EtOH)]; ${ }^{1} \mathrm{H}$ NMR $\left(300 \mathrm{MHz}, \mathrm{CDCl}_{3}\right): \delta 0.84(\mathrm{t}, J=7.3 \mathrm{~Hz}, 3 \mathrm{H})$, 1.20-1.95 (m, 10H), 2.65-2.94 (m, 2H), 3.23-3.42 (m, 1H), $9.10(\mathrm{~s}$, br, $1 \mathrm{H}), 9.34(\mathrm{~s}, \mathrm{br}$, $1 \mathrm{H}) ;{ }^{13} \mathrm{C}$ NMR $\left(75 \mathrm{MHz}, \mathrm{CDCl}_{3}\right): \delta 57.0,44.6,35.2,28.0,22.3,22.0,18.4,13.6$; Enantiomeric excess was determined by HPLC analysis of its $p$-toluenesulfonamide: (hexane $/ i-\operatorname{PrOH}=99 / 1$, flow rate $=1 \mathrm{~mL} / \mathrm{min}), \mathrm{t}_{\mathrm{R}}=25.0 \min (S), \mathrm{t}_{\mathrm{R}}=27.1 \mathrm{~min}(R)$; $[\alpha]_{589}^{25}-34.5$ (c 1.0, benzene) [lit. ${ }^{7}$ (for $S$ enantiomer): $[\alpha]_{589}^{24}+39.6$ (c 0.53 , benzene)]; IR (neat): 3063, 3021, 2937, 2871, 1598, 1337, 1151, 1093, 932, 815, 712, 694, 653, 599, $552 \mathrm{~cm}^{-1} .{ }^{1} \mathrm{H}$ NMR $\left(300 \mathrm{MHz}, \mathrm{CDCl}_{3}\right): \delta 0.86(\mathrm{t}, J=7.3 \mathrm{~Hz}, 3 \mathrm{H}), 1.10-1.65(\mathrm{~m}, 10 \mathrm{H})$, 2.39 (s, 3H), $2.96(\mathrm{t}, J=13.5 \mathrm{~Hz}, 1 \mathrm{H}), 3.71(\mathrm{dd}, J=3.7 \mathrm{~Hz}, 13.5 \mathrm{~Hz}, 1 \mathrm{H}), 3.95-4.06(\mathrm{~m}$, 1H), $7.25(\mathrm{~d}, J=7.8 \mathrm{~Hz}, 2 \mathrm{H}), 7.69(\mathrm{~d}, J=7.8 \mathrm{~Hz}, 2 \mathrm{H}) ;{ }^{13} \mathrm{C} \mathrm{NMR}\left(75 \mathrm{MHz}, \mathrm{CDCl}_{3}\right): \delta$ $143.2,139.3,129.5,126.9,52.7,40.5,31.6,27.3,24.4,21.4,19.6,18.4,13.9$; MS (CI) $m / z$ (relative intensity): $282\left(\mathrm{M}^{+}, 100\right), 238\left(\mathrm{M}^{+}-\mathrm{CH}_{2} \mathrm{CH}_{2} \mathrm{CH}_{3}, 31\right)$. 
one (6).

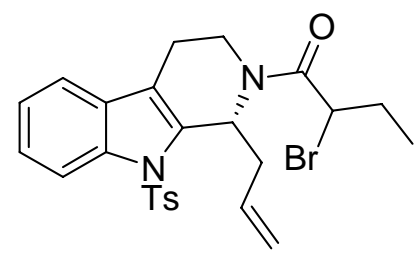

A solution of amine $3 \mathbf{g}(220 \mathrm{mg}, 0.60 \mathrm{mmol})$ in $\mathrm{CH}_{2} \mathrm{Cl}_{2}(20 \mathrm{~mL})$ was treated with DCC (1.18 g, $5.72 \mathrm{mmol}), 2$-bromobutyric acid (957 mg, $5.73 \mathrm{mmol})$ and DMAP (20 mg) at $10{ }^{\circ} \mathrm{C}$. The reaction was allowed to warm to room temperature for 2 hours. Solid material was filtered off and the filtrate was washed with $10 \% \mathrm{NaHCO}_{3}$ (aq.). Removal of the volatiles gave the crude amide 6 in quantitative yield. This crude material was used without further purification.

(E)-Ethyl 4-((R)-2-(2-bromobutanoyl)-9-tosyl-2,3,4,9-tetrahydro-1H-pyrido[3,4b]indol-1-yl)but-2-enoate (7).

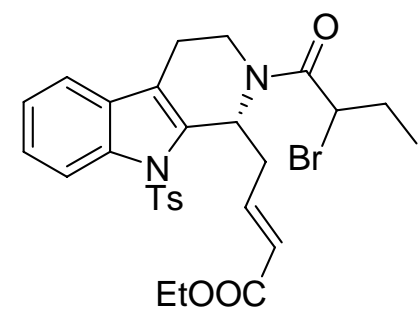

The resulting compound 6 was dissolved in dioxane $/ \mathrm{H}_{2} \mathrm{O}(3: 1,15 \mathrm{~mL}) .2,6$-Lutidine $(0.15$ $\mathrm{mL}), \mathrm{OsO}_{4}(0.6 \mathrm{~mL}, 0.022 \mathrm{M}$ in water $)$ and $\mathrm{NaIO}_{4}(510 \mathrm{mg}, 4.68 \mathrm{mmol})$ were added and the mixture was stirred at room temperature for 4 hours. The reaction mixture was then diluted with EtOAc $(10 \mathrm{~mL})$ and water $(10 \mathrm{~mL})$. The organic phase was separated and the aqueous phase was washed with EtOAc $(3 \times 10 \mathrm{~mL})$. The combined organic phases 
were washed with saturated $\mathrm{Na}_{2} \mathrm{SO}_{3}$ and brine then dried over $\mathrm{Na}_{2} \mathrm{SO}_{4}$. Following removal of the solvent, the crude aldehyde was taken up in $\mathrm{CH}_{2} \mathrm{Cl}_{2}(10 \mathrm{~mL})$ and treated with $\mathrm{Ph}_{3} \mathrm{P}=\mathrm{CHCOOEt}(0.28 \mathrm{~g}, 0.8 \mathrm{mmol})$ at room temperature for 2 hours. Solvent was then removed under reduced pressure. Flash column chromatography (2:1 EtOAc/hexane) afforded the title compound ( $288 \mathrm{mg}, 82 \%$ yield for three steps). ${ }^{1} \mathrm{H}$ NMR (300 MHz, DMSO, mixture of diastereomers and rotamers) $\delta 0.75-0.89(\mathrm{~m}, 3 \mathrm{H}), 1.15(\mathrm{t}, J=7.1 \mathrm{~Hz}$, $3 \mathrm{H}), 1.79-2.26(\mathrm{~m}, 5 \mathrm{H}), 2.57-3.23(\mathrm{~m}, 4 \mathrm{H}), 3.43-3.61(\mathrm{~m}, 1 \mathrm{H}), 3.94-4.68(\mathrm{~m}, 2 \mathrm{H}), 4.76-$ $5.64(\mathrm{~m}, 1 \mathrm{H})$, 5.81-7.08 (m, 3H), 7.15-7.48 (m, 5H), 7.55-7.69 (m, 1.74H), 7.74-7.81 (m, $0.26 \mathrm{H}), 7.94(\mathrm{~d}, J=8.4 \mathrm{~Hz}, 0.13 \mathrm{H}), 8.01(\mathrm{~d}, J=8.2 \mathrm{~Hz}, 0.87 \mathrm{H}) ;{ }^{13} \mathrm{C}$ NMR $(75 \mathrm{MHz}$, $\mathrm{CHCl}_{3}$, signals for the major isomer) $167.8,165.9,144.9,144.0,136.7,134.8,133.9$, 129.7, 129.6, 129.3, 126.7, 125.0, 124.1, 118.5, 118.0, 115.4, 67.0, 60.1, 48.8, 44.7, 37.4, 28.8, 21.5, 20.4, 14.2, 12.1; MS (ESI) $m / z$ (relative intensity): $558\left(\mathrm{M}^{+}+\mathrm{H}, 100\right), 238$ $\left(\mathrm{M}^{+}-\mathrm{CH}_{2} \mathrm{CH}_{2} \mathrm{CH}_{3}, 31\right)$; HRMS $m / z$ Calcd. for $\mathrm{C}_{28} \mathrm{H}_{32} \mathrm{BrN}_{2} \mathrm{O}_{5} \mathrm{~S}\left(\mathrm{M}^{+}+\mathrm{H}\right)$ : 587.1215. Found: 587.1207. 
Ethyl 2-((2S,3R,12bR)-3-ethyl-4-oxo-12-tosyl-1,2,3,4,6,7,12,12b-octahydroindolo[2,3a]quinolizin-2-yl)acetate (8).

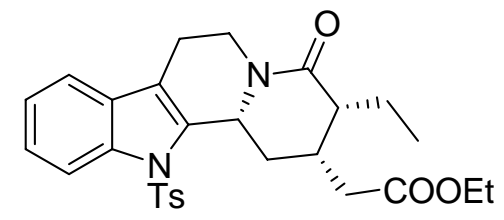

To a solution of compound $7(220 \mathrm{mg}, 0.375 \mathrm{mmol})$ in $\mathrm{THF}(35 \mathrm{~mL})$ at $-78{ }^{\circ} \mathrm{C}$ was added $n$-BuLi (0.414 mmol, $0.28 \mathrm{~mL}, 1.48 \mathrm{M}$ in hexane) dropwise. The resulting clear solution was stirred at that temperature for an additional 2 minutes. The reaction was quenched with a minimum amount of saturated $\mathrm{NH}_{4} \mathrm{Cl}$ solution. Evaporation of the solvent gave an oily residue that was purified by flash column chromatography (2:1 EtOAc/hexane) to afford the title compound (8) as a single diastereomer (119 mg, 63\%). ${ }^{1} \mathrm{H}$ NMR (300 $\left.\mathrm{MHz}, \mathrm{CDCl}_{3}\right): \delta 0.98(\mathrm{t}, J=7.4 \mathrm{~Hz}, 3 \mathrm{H}), 1.18(\mathrm{t}, J=7.1 \mathrm{~Hz}, 3 \mathrm{H}), 1.45-1.81(\mathrm{~m}, 3 \mathrm{H}), 2.12$ (dd, $J=9.4 \mathrm{~Hz}, 15.5 \mathrm{~Hz}, 1 \mathrm{H}), 2.23(\mathrm{~s}, 3 \mathrm{H}), 2.38$ (dd, $J=5.7 \mathrm{~Hz}, 15.5 \mathrm{~Hz}, 1 \mathrm{H}), 2.45-2.81$ (m, 5H), $3.05(\mathrm{dt}, J=13.5 \mathrm{~Hz}, 5.2 \mathrm{~Hz}, 1 \mathrm{H}), 4.01-4.12(\mathrm{~m}, 2 \mathrm{H}), 4.96-5.11(\mathrm{~m}, 2 \mathrm{H}), 7.04$ (d, $J=8.2 \mathrm{~Hz}, 2 \mathrm{H}), 7.15-7.32(\mathrm{~m}, 3 \mathrm{H}), 7.41(\mathrm{~d}, J=8.2 \mathrm{~Hz}, 2 \mathrm{H}), 8.06(\mathrm{~d}, J=9.0 \mathrm{~Hz}, 1 \mathrm{H})$; ${ }^{13} \mathrm{C}$ NMR $\left(75 \mathrm{MHz}, \mathrm{CDCl}_{3}\right): \delta 172.5,172.0,144.9,138.1,135.7,133.2,130.2,129.2$, $126.4,125.2,124.5,123.1,118.6,116.3,60.5,53.7,45.3,38.5,36.9,35.6,31.1,21.7$, $21.4,20.7,14.1,12.8$.

ent-Corynantheidol (9).

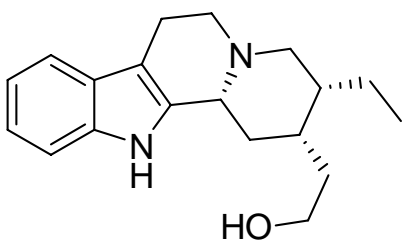


Ester 8 (110 mg, $0.216 \mathrm{mmol}$ ) was dissolved in THF (35 mL). LAH (130 mg, 15 equiv.) was added in 4 portions during the course of 2 hours. The suspension was stirred at ambient temperature over night. The reaction was then cooled to $0{ }^{\circ} \mathrm{C}$ and unreacted LAH quenched carefully with saturated $\mathrm{NH}_{4} \mathrm{Cl}$. Removal of solid by filtration resulted in a clear solution that was concentrated to an oily residue. Purification by flash column chromatography (12:1 $\left.\mathrm{CH}_{2} \mathrm{Cl}_{2} / \mathrm{MeOH}, 0.5 \% \mathrm{NH}_{4} \mathrm{OH}\right)$ gave the desired compound (9) as a white solid (57 mg, 88\%), which has spectroscopic properties identical to those reported in the literature. $[\alpha]_{\mathrm{D}}{ }^{20}+93.2$ (c 0.65 , pyridine) $\left[\right.$ lit. $^{8}$ for corynantheidol: $[\alpha]_{\mathrm{D}}{ }^{20}-$ 93.0 (c 0.52 , pyridine)]; ${ }^{1} \mathrm{H}$ NMR (300 MHz, $\left.\mathrm{CDCl}_{3}\right): \delta 0.92(\mathrm{t}, J=7.3 \mathrm{~Hz}, 3 \mathrm{H}), 1.17-$ $1.94(\mathrm{~m}, 8 \mathrm{H}), 2.24(\mathrm{~s}, \mathrm{br}, 1 \mathrm{H}), 2.32(\mathrm{dd}, J=2.2 \mathrm{~Hz}, 11.5 \mathrm{~Hz}, 1 \mathrm{H}), 2.46-2.75(\mathrm{~m}, 2 \mathrm{H})$, 2.86-3.15 (m, 4H), $3.71(\mathrm{dt}, J=2.5 \mathrm{~Hz}, 6.5 \mathrm{~Hz}, 2 \mathrm{H}), 7.04-7.15(\mathrm{~m}, 2 \mathrm{H}), 7.28$ (d, $J=7.4$ Hz, 1H), 7.45 (d, $J=7.3 \mathrm{~Hz}, 1 \mathrm{H}), 8.05$ (s, br, 1H). MS (EI) $\mathrm{m} / z$ (relative intensity): 298 $\left(\mathrm{M}^{+}\right.$, 85), $297\left(\mathrm{M}^{+}-\mathrm{H}, 100\right)$; HRMS $m / z$ Calcd. for $\mathrm{C}_{19} \mathrm{H}_{25} \mathrm{NO}_{2}\left(\mathrm{M}^{+}-\mathrm{H}\right)$ : 297.1967. Found: 297.1974.

\section{References}

${ }^{1}$ Wu, T. R.; Shen, L.; Chong, J. M. Org. Lett. 2002, 6, 2701.

${ }^{2}$ (a) For syntheses of electron rich 3,4-dihydroisoquinolines, see: Rohloff, J. C.; Dyson, N. H.; Gardner, J. O.; Alfredson, T. V.; Sparacino, M. L.; Robinson, J. J. Org. Chem. 1993, 58, 1935. (b) For syntheses of electron poor 3,4-dihydroisoquinolines, see: Pelletier, J. C.; Cava, M. P. J. Org. Chem. 1987, 52, 616. (c) For the synthesis of 3,4-dihydro- $\beta$ carboline, see: Bertrand, M.; Poissonnet, G.; Thérét-Bettiol, M.; Gaspard, C.; Werner, G. H.; Pfeiffer, B.; Renard, P.; Leonce, S.; Dodd, R. H. Bioorg. Med. Chem. 2001, 9, 2155. (d) For the synthesis of 3,4-dihydro-9-( $p$-toluenesulfonyl)- $\beta$-carboline, see: Rey, A. W.; 
Szarek, W. A.; Maclean, D. B. Can. J. Chem. 1992, 70, 2922. (e) For the synthesis of 1pyrroline, see: Ochiai, M.; Inenaga, M.; Nagao, Y.; Moriarty, R. M.; Vaid, R. K.; Duncan, M. P. Tetrahedron Lett. 1988, 29, 6917. (f) For the synthesis of $\Delta^{1}$-piperideine, see: Scully, F. E. J. Org. Chem. 1980, 45, 1515.

${ }^{3}$ Nakamura, M.; Hirai, A.; Makamura E. J. Am. Chem. Soc. 1996, $118,8489$.

${ }^{4}$ Park, S. H.; Kang, H. J.; Ko, S.; Park, S.; Chang, S. Tetrahedron: Asymmetry 2001, 12, 2621.

${ }^{5}$ Szawkalo, J.; Zawadzka, A.; Wojtasiewicz, K.; Leniewski, A.; Drabowicz, J.; Czarnocki, Z. Tetrahedron: Asymmetry 2005, 16, 3619.

${ }^{6}$ Amat, M.; Llor, N.; Hidalgo, J.; Escolano, C.; Bosch, J. J. Org. Chem. 2003, 68, 1919.

${ }^{7}$ Gallagher, T.; Eskici, M. Synlett 2000, 1368.

${ }^{8}$ Beard, R. L.; Meyers, A. I. J. Org. Chem. 1991, 56, 2091. 


\section{${ }^{1}$ H NMR spectrum of compound $3 c$.}
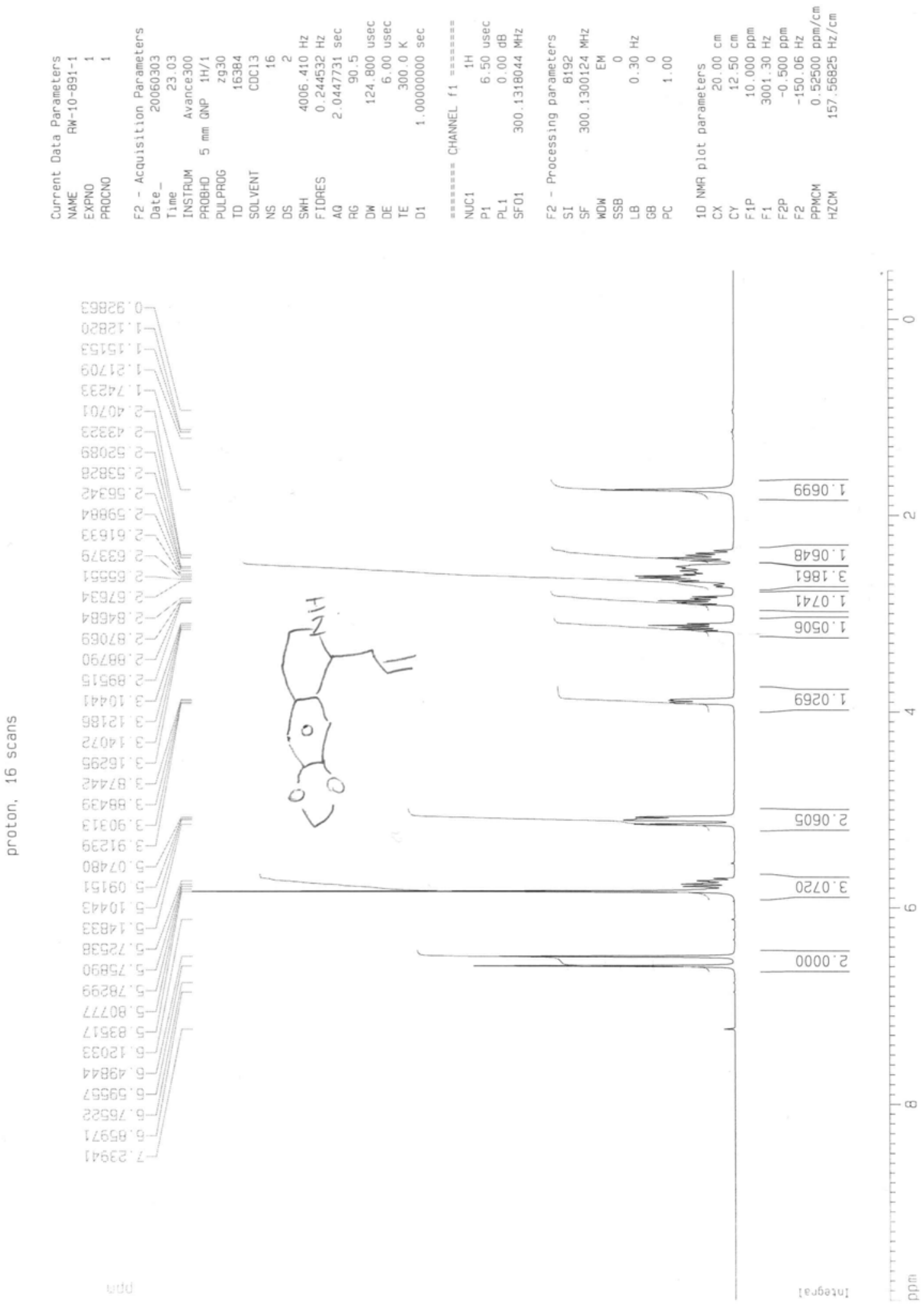
${ }^{13} \mathrm{C}$ NMR spectrum of compound $3 c$.
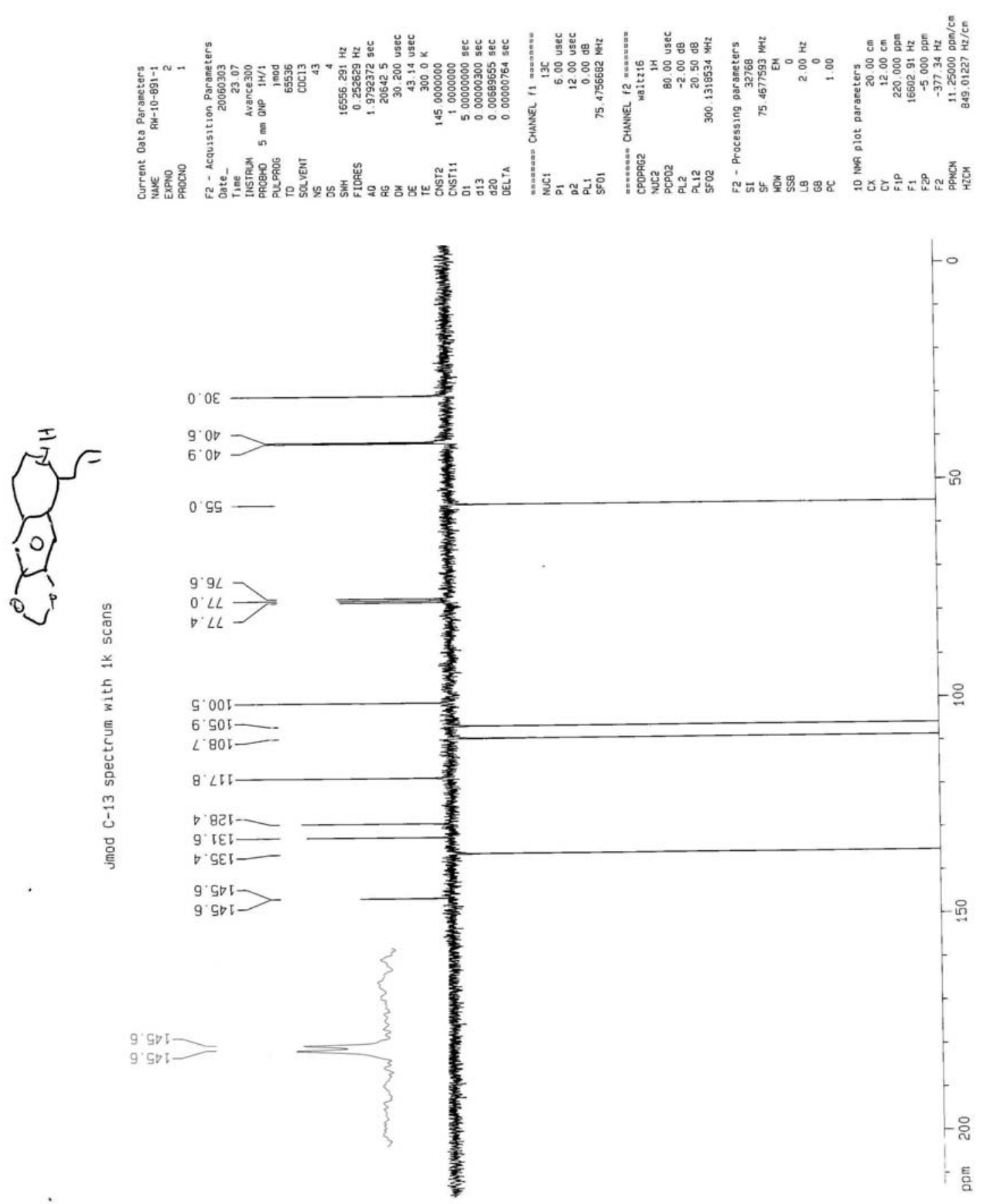
${ }^{1} \mathrm{H}$ NMR spectrum of the trifluoroacetamide of compound 3c.
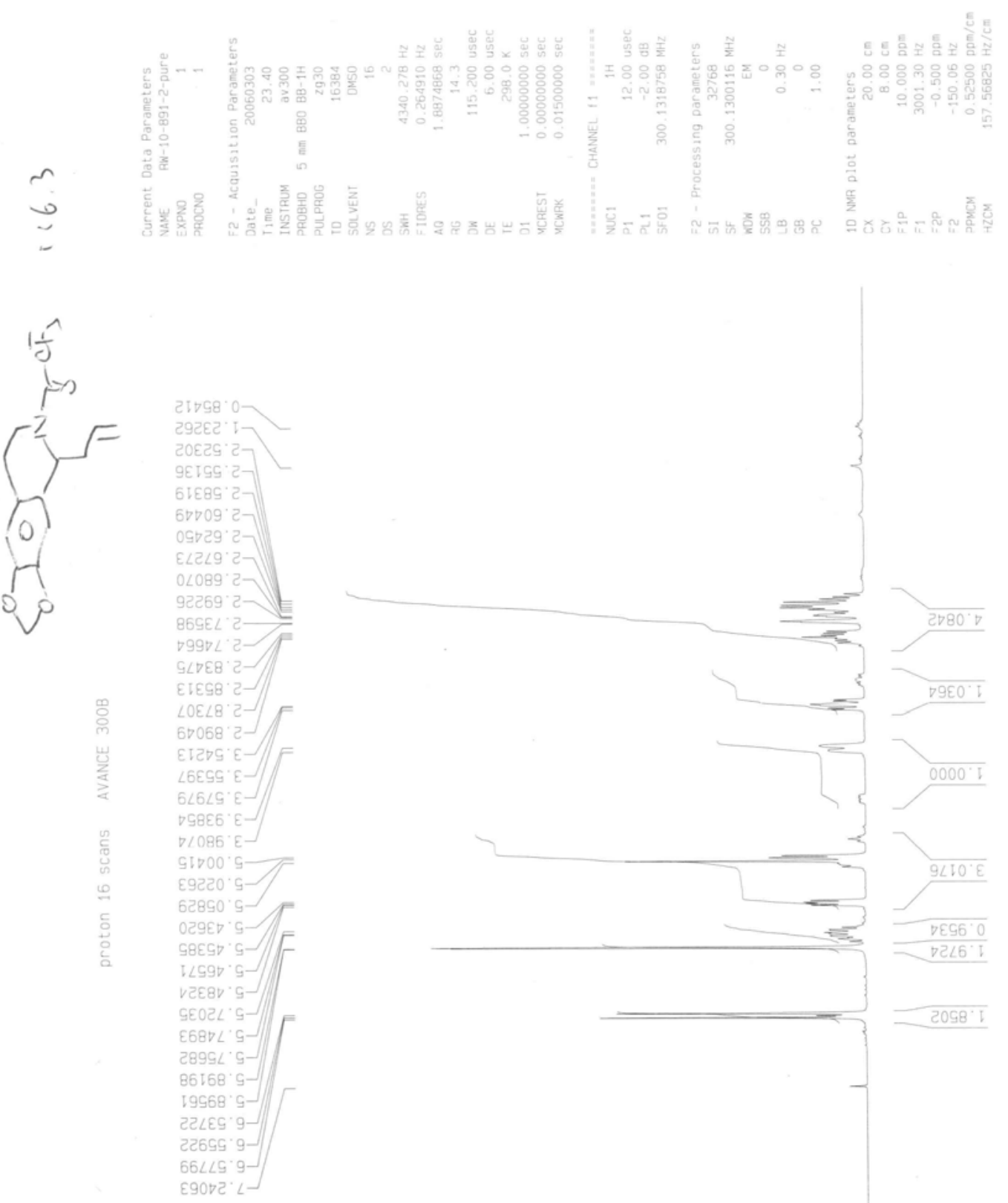
${ }^{13} \mathrm{C}$ NMR spectrum of the trifluoroacetamide of compound 3c.
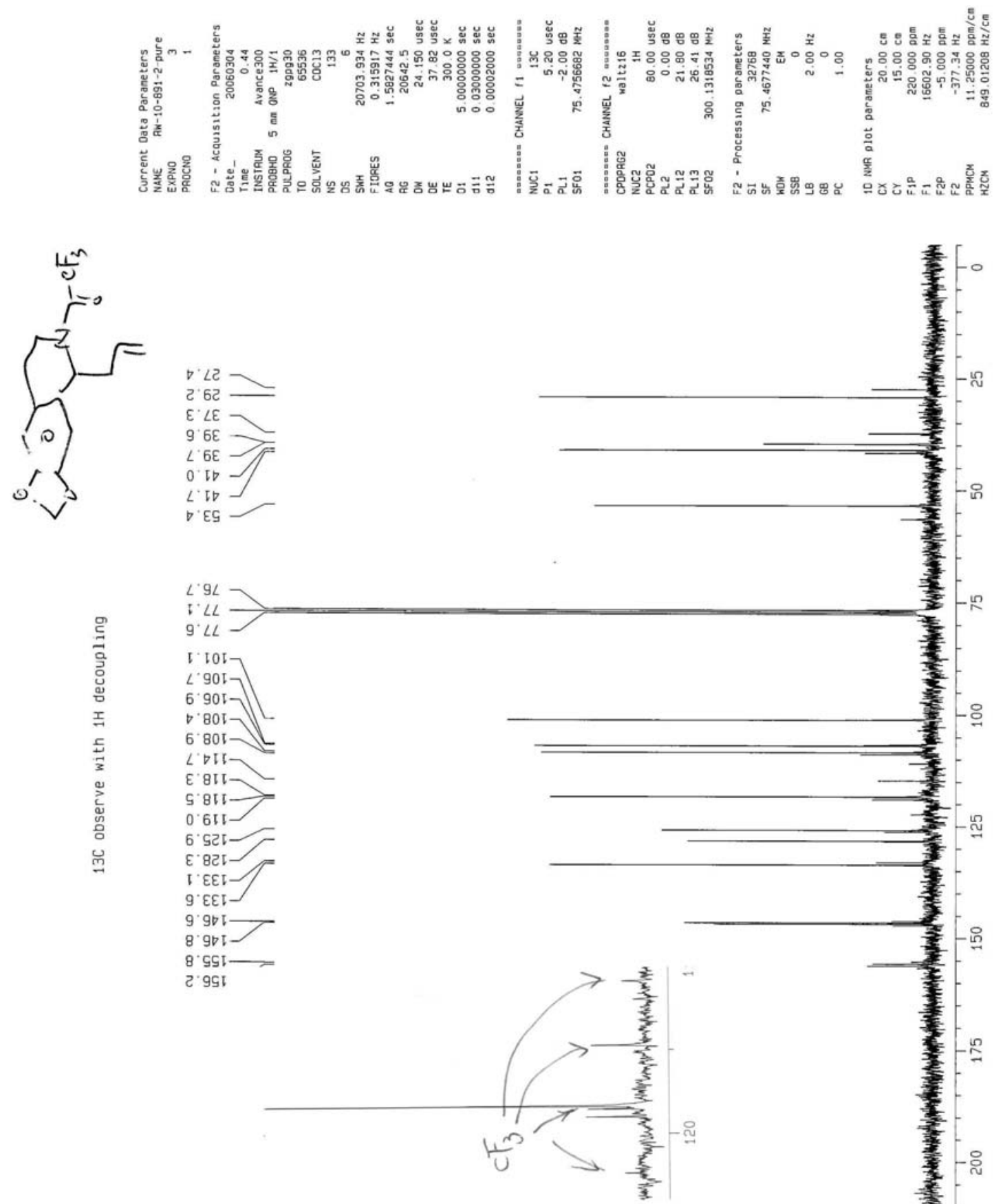


\section{${ }^{1} \mathrm{H}$ NMR spectrum of compound $3 \mathrm{~d}$.}
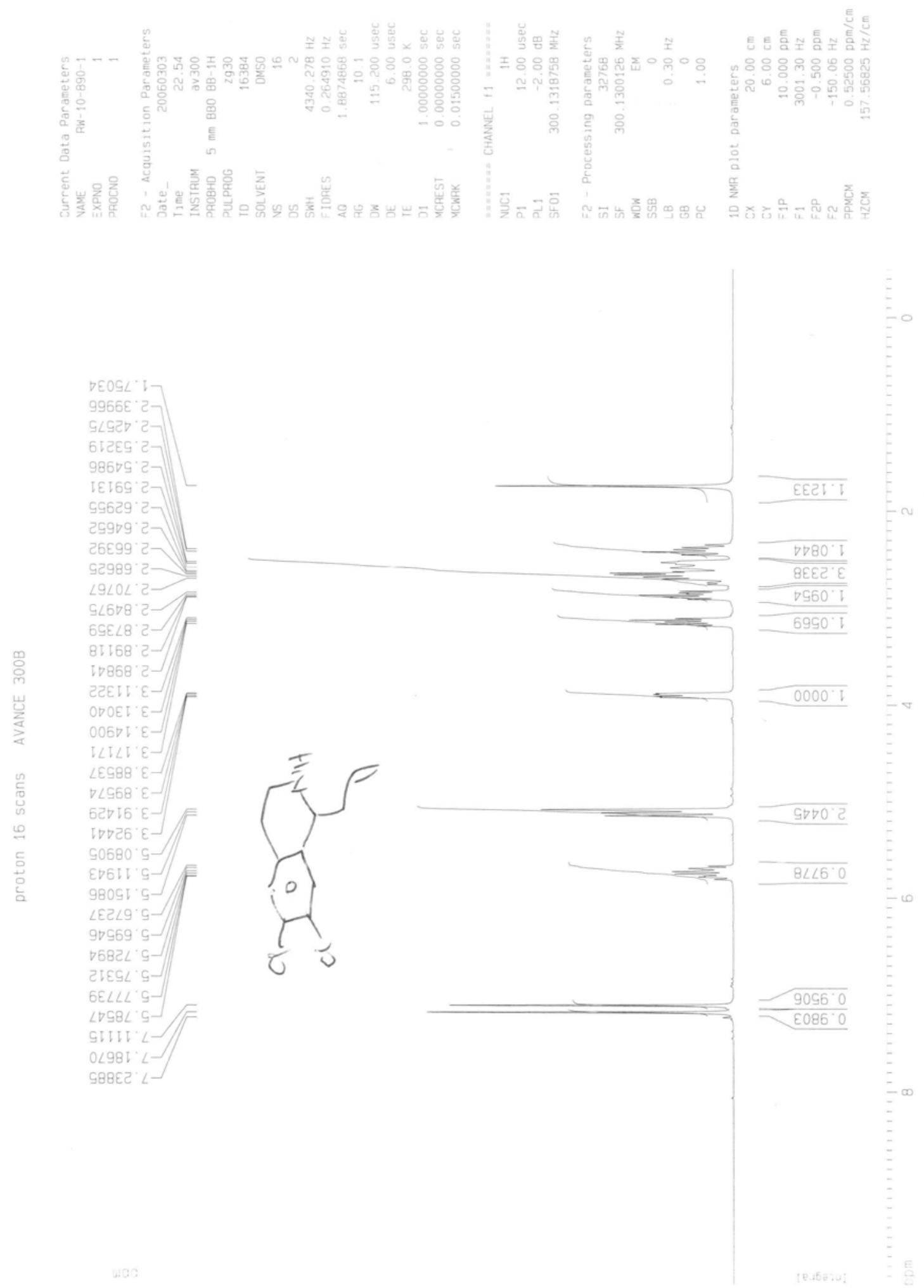


\section{${ }^{13} \mathrm{C}$ NMR spectrum of compound 3d.}
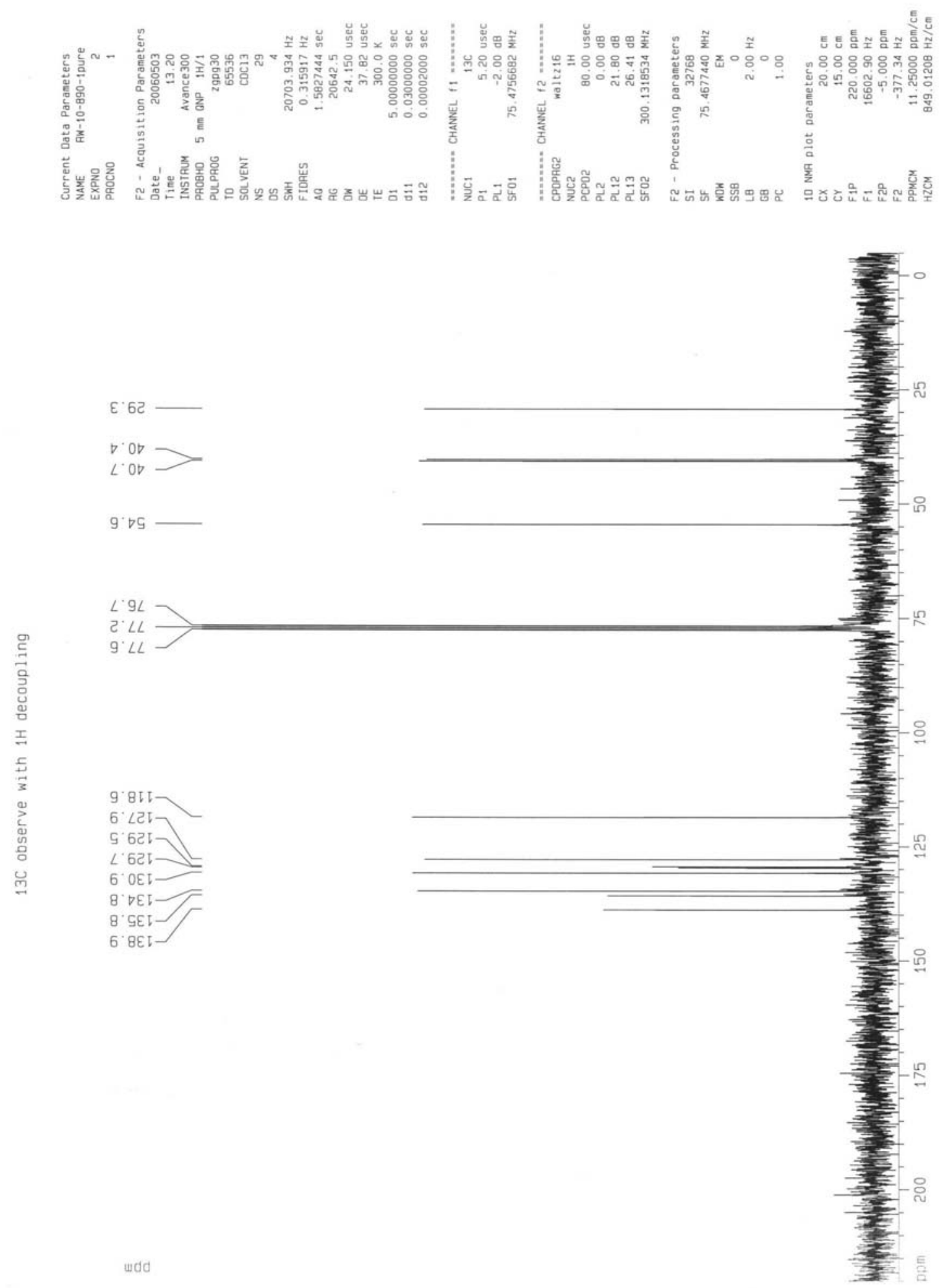


\section{${ }^{1} \mathrm{H}$ NMR spectrum of compound $3 \mathrm{e}$.}
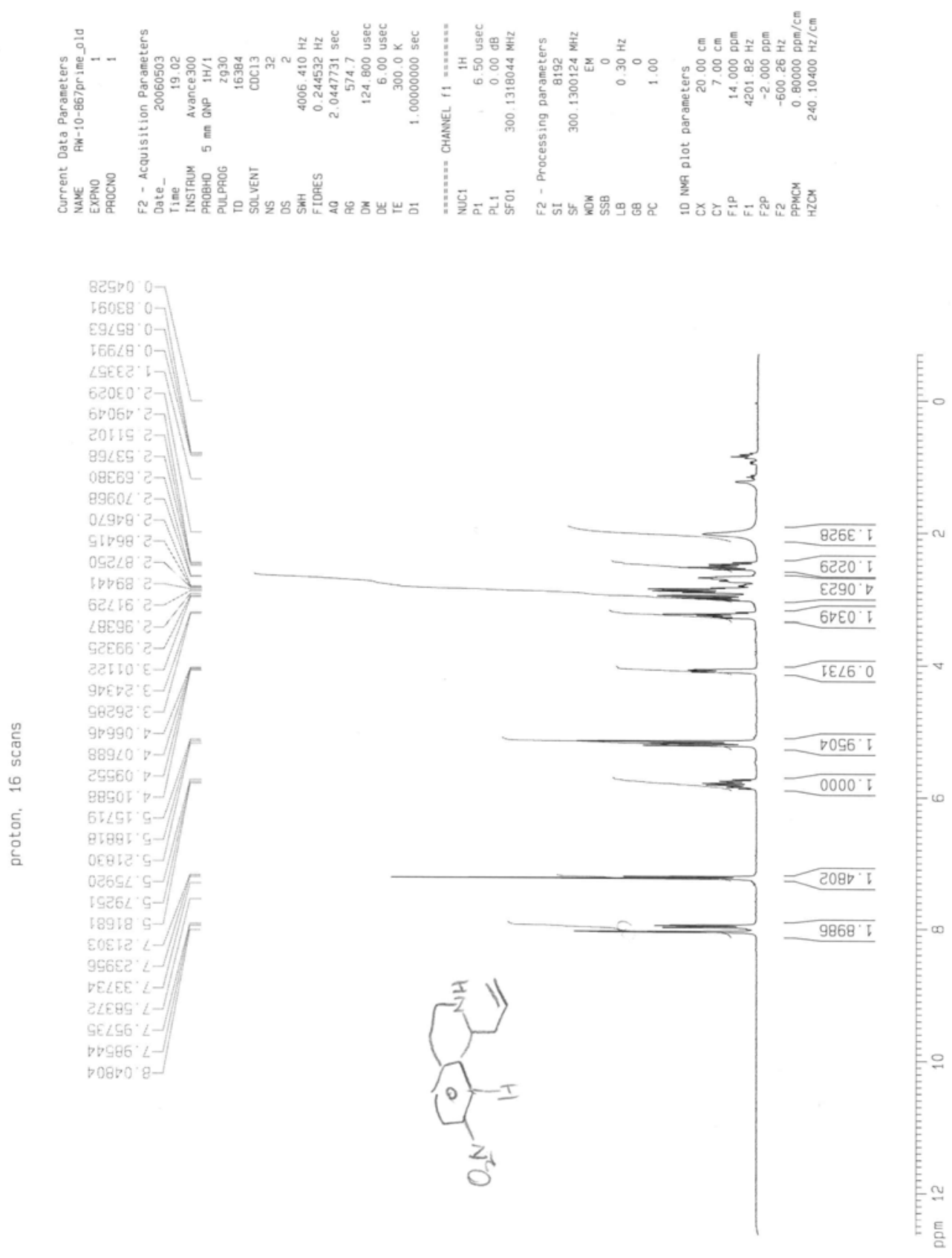


\section{${ }^{13} \mathrm{C}$ NMR spectrum of compound $3 e$.}
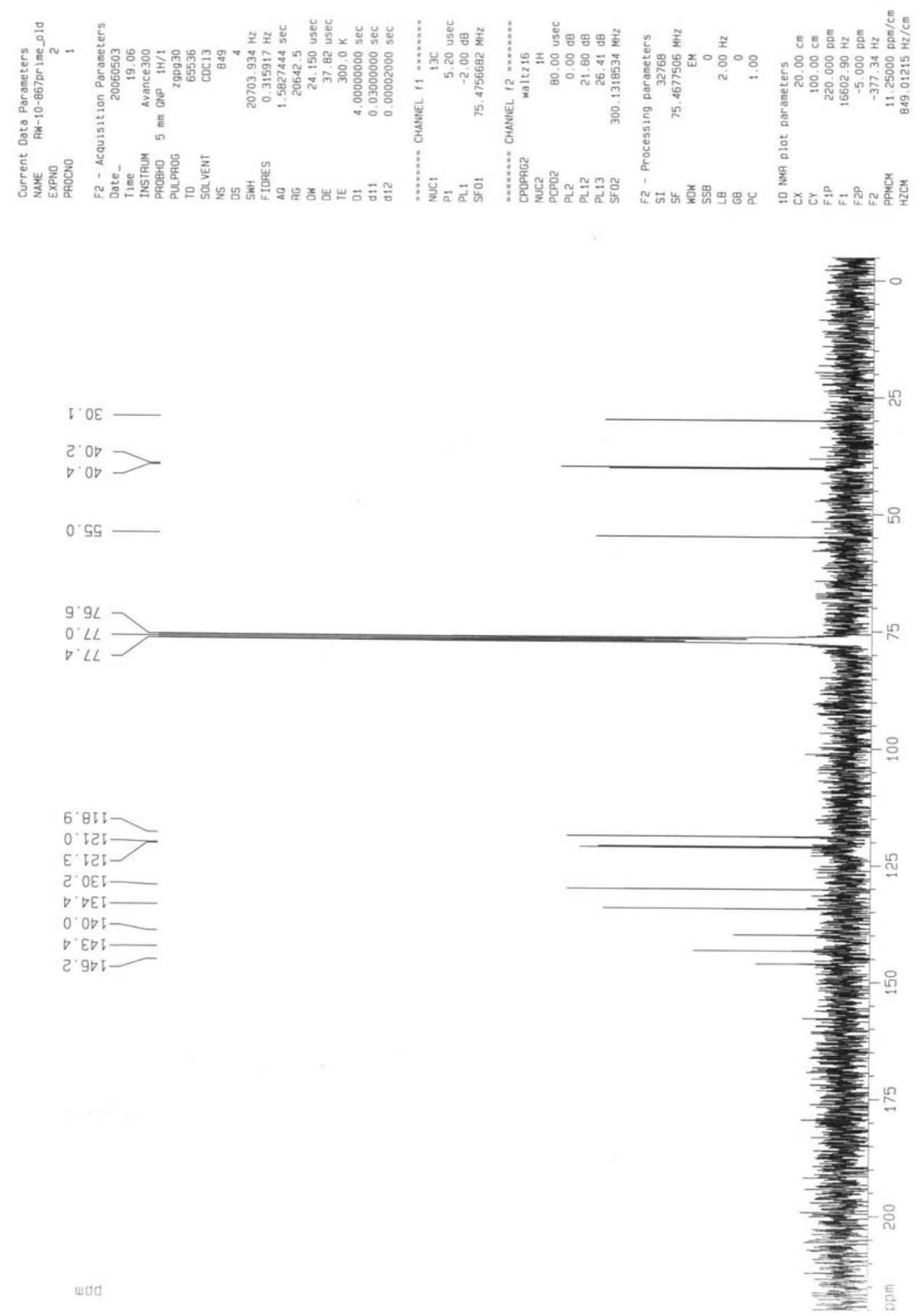
${ }^{1} \mathrm{H}$ NMR spectrum of the trifluoroacetamide of compound 3 .
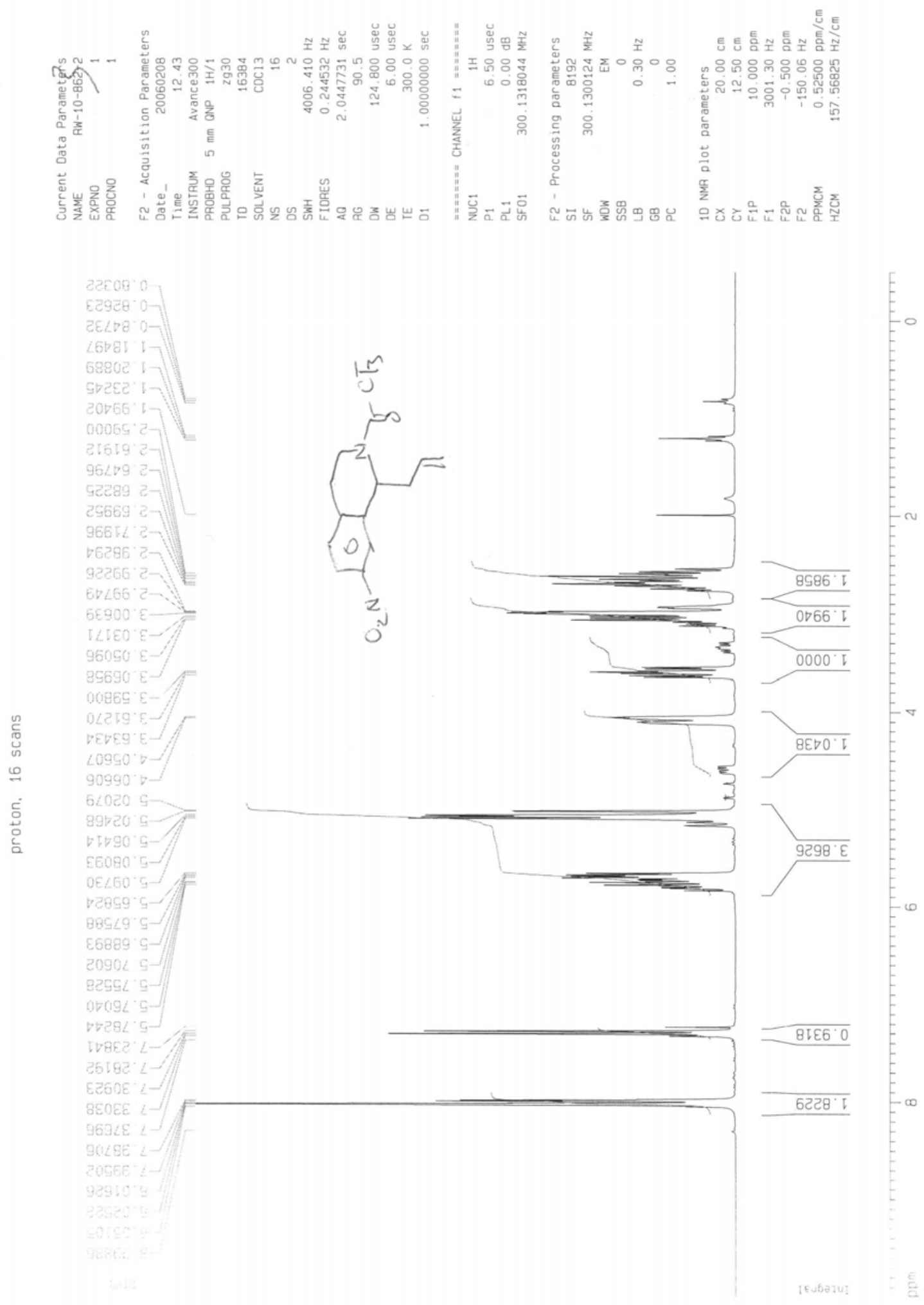
${ }^{13} \mathrm{C}$ NMR spectrum of the trifluoroacetamide of compound $3 e$.
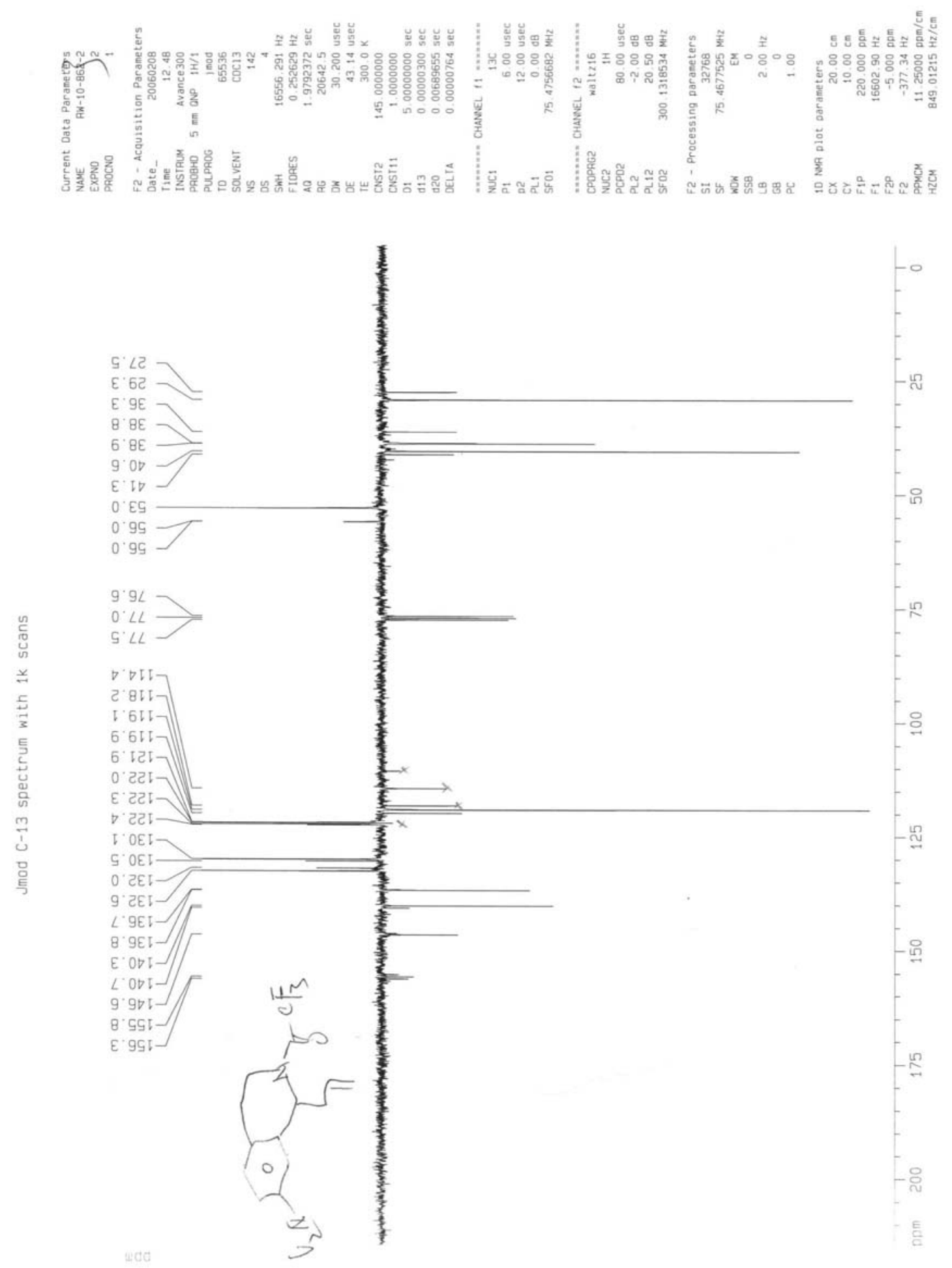


\section{${ }^{1}$ H NMR spectrum of compound $3 g$.}
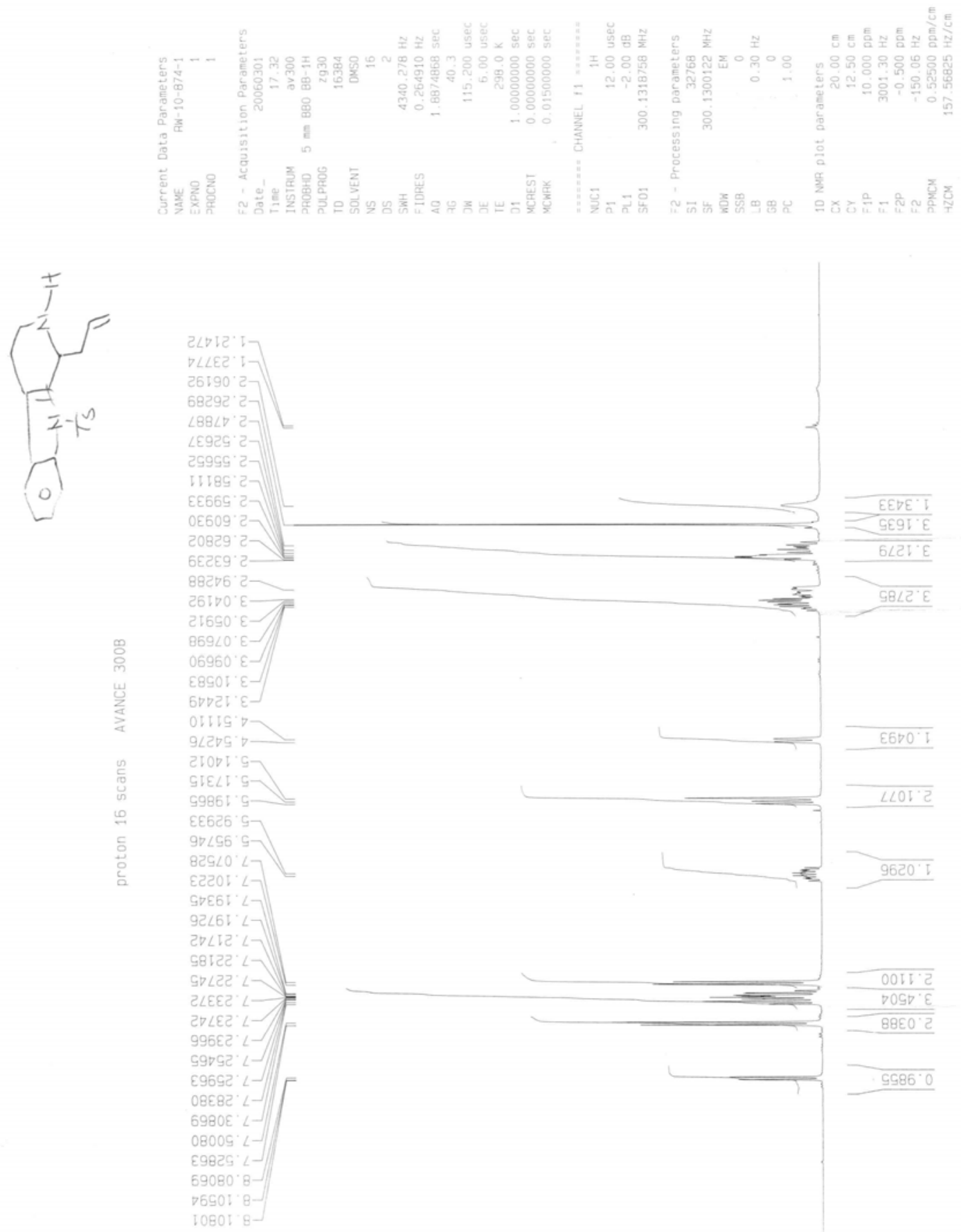


$$
4
$$




\section{${ }^{1} \mathrm{H}$ NMR spectrum of the trifluoroacetamide of compound $3 g$.}
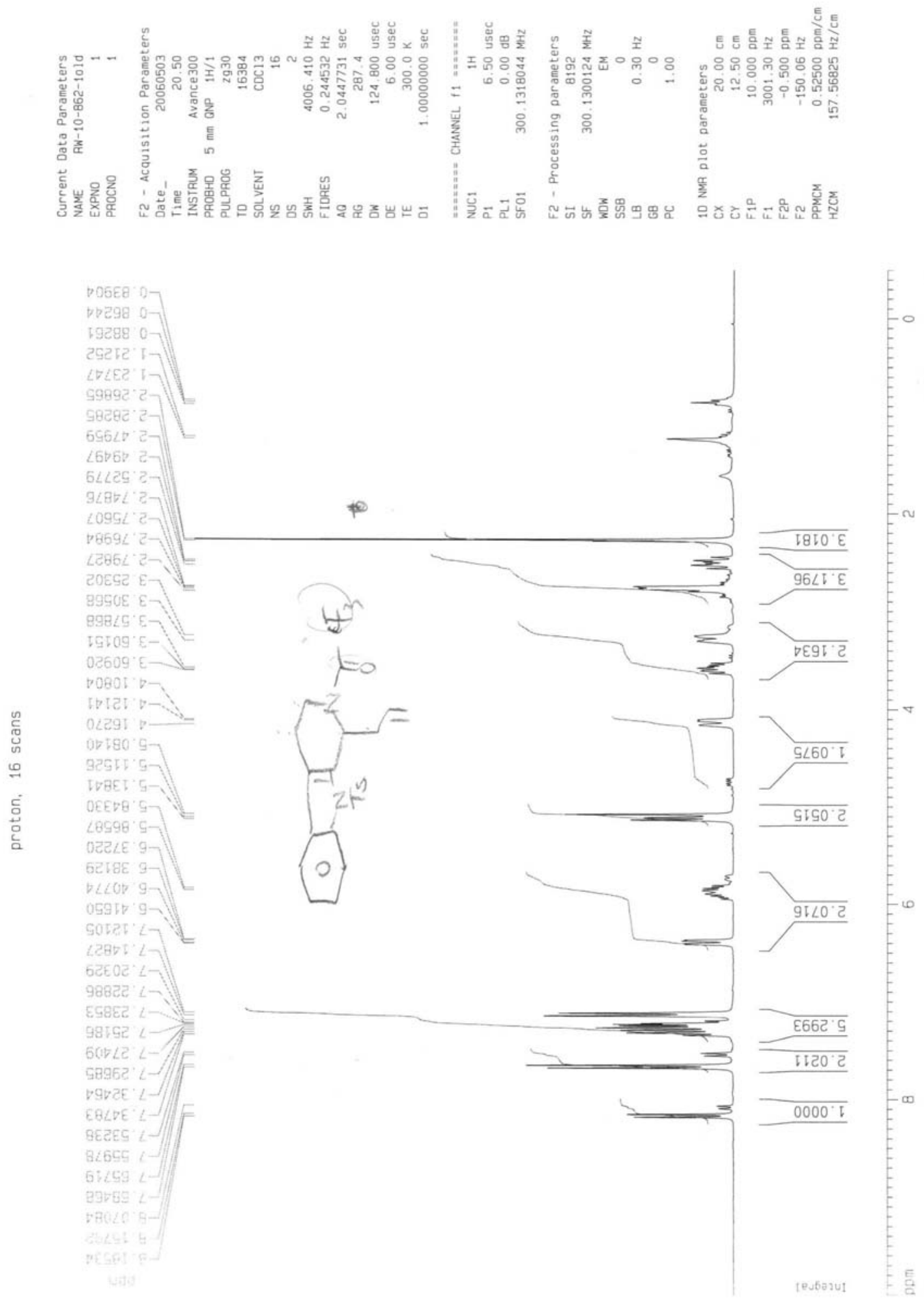


\section{${ }^{13} \mathrm{C}$ NMR spectrum of the trifluoroacetamide of compound $3 g$.}
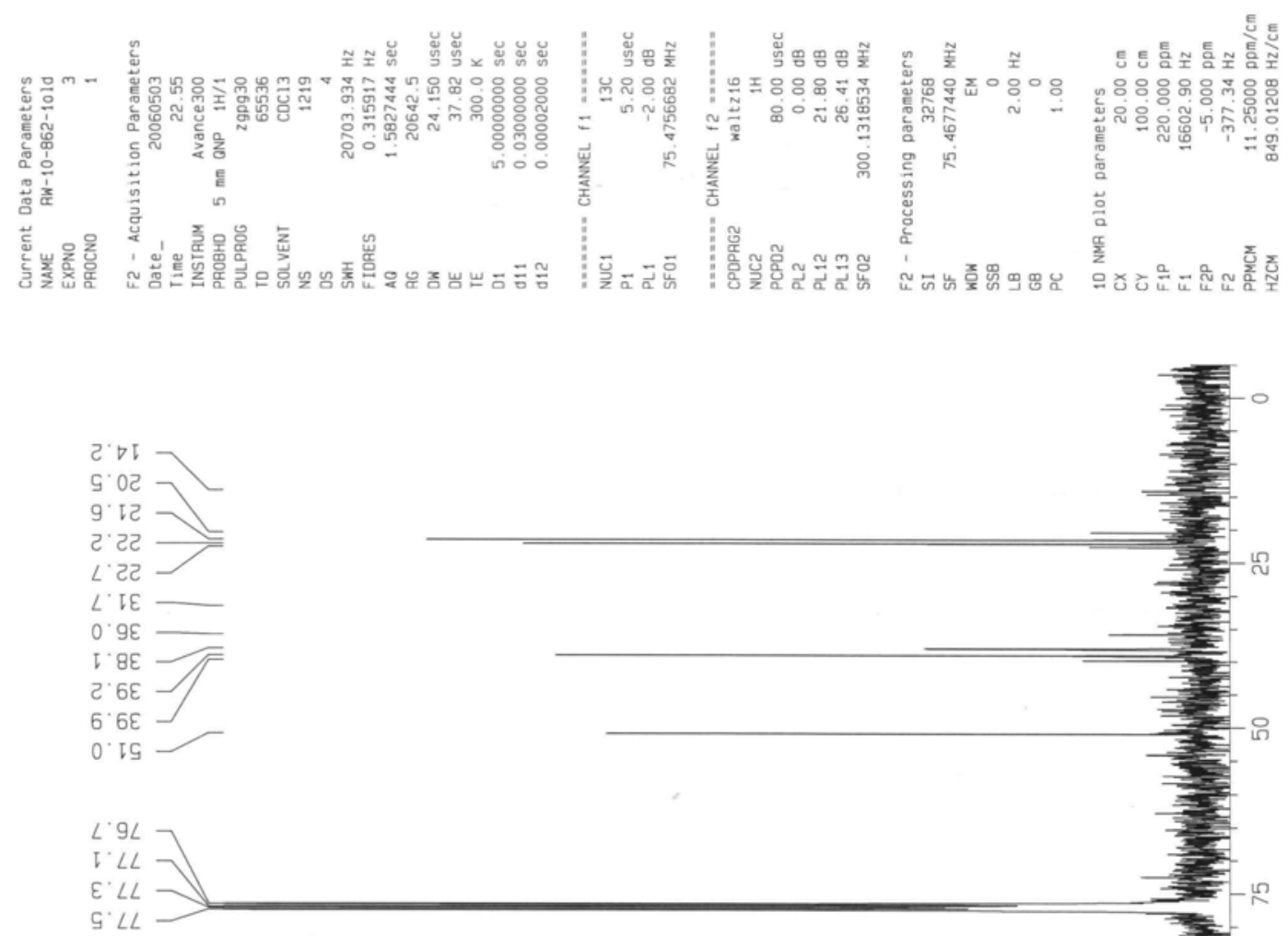

E SI

g. GI -

$[\cdot 8[1]$

g. 817

8.815

c.

$\varepsilon \cdot \mathrm{c}$ เा一-

G. $92 \mathrm{c}$

8. $251-7$

2. $255-1-15$

$6.6 \mathrm{CL}-1$

$0.0 E[-$

L. $E \varepsilon \mathrm{LI}-$

$0 . \triangle E[-T$

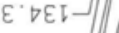

$[\cdot g E[-1$

C. $\mathrm{CL}_{\mathrm{L}} \mathrm{L}-1$

$\varepsilon \cdot G \nabla[-1$

ह. 99 -

8 . 99

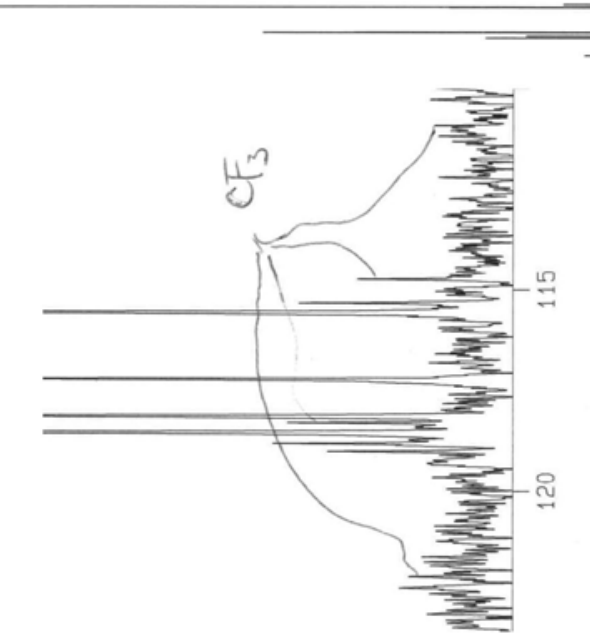


${ }^{1}$ H NMR of (R)-3-(6,7-dimethoxy-1,2,3,4-tetrahydroisoquinolin-1-yl)propan-1-ol
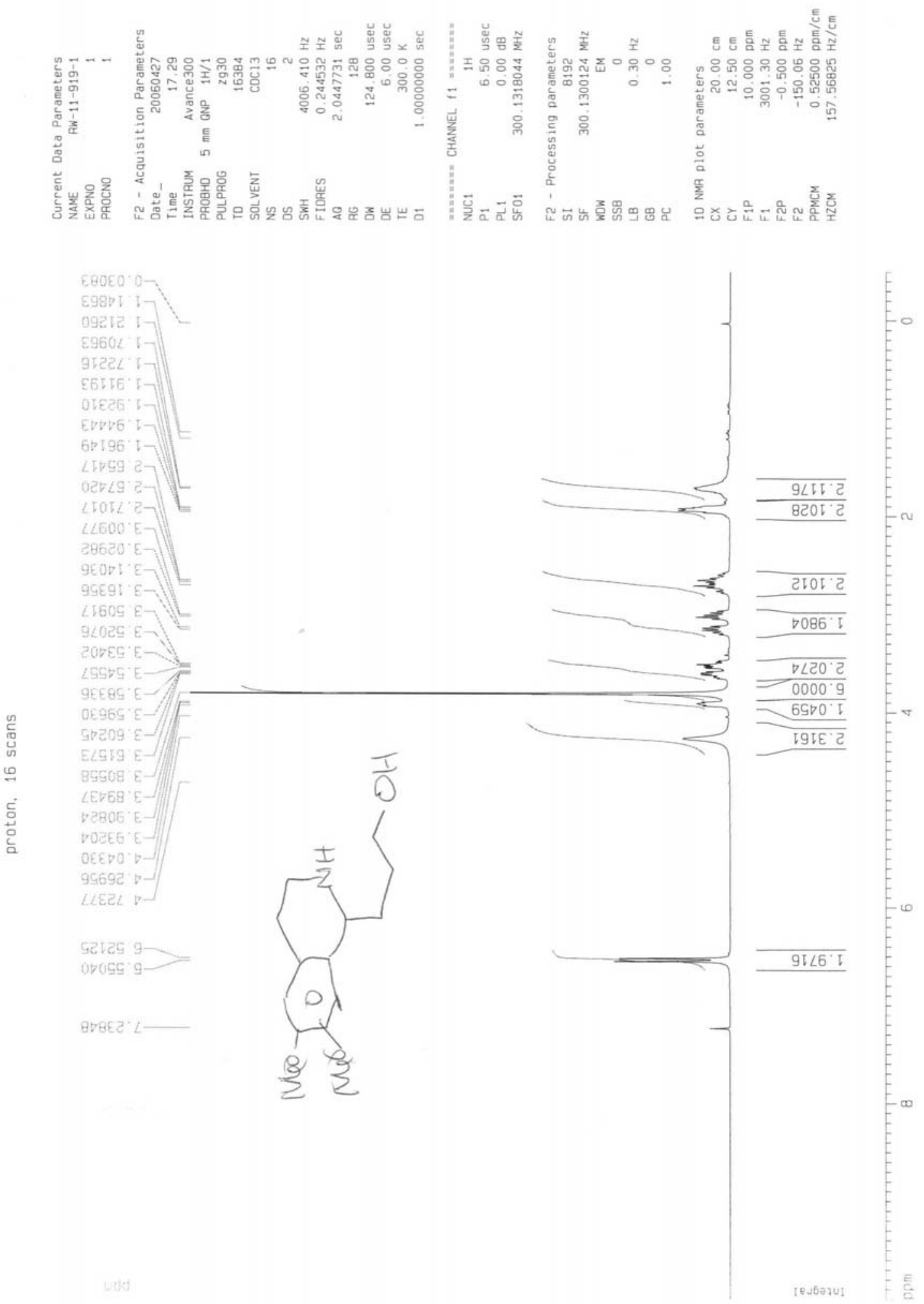
${ }^{13}$ C NMR of (R)-3-(6,7-dimethoxy-1,2,3,4-tetrahydroisoquinolin-1-yl)propan-1-ol
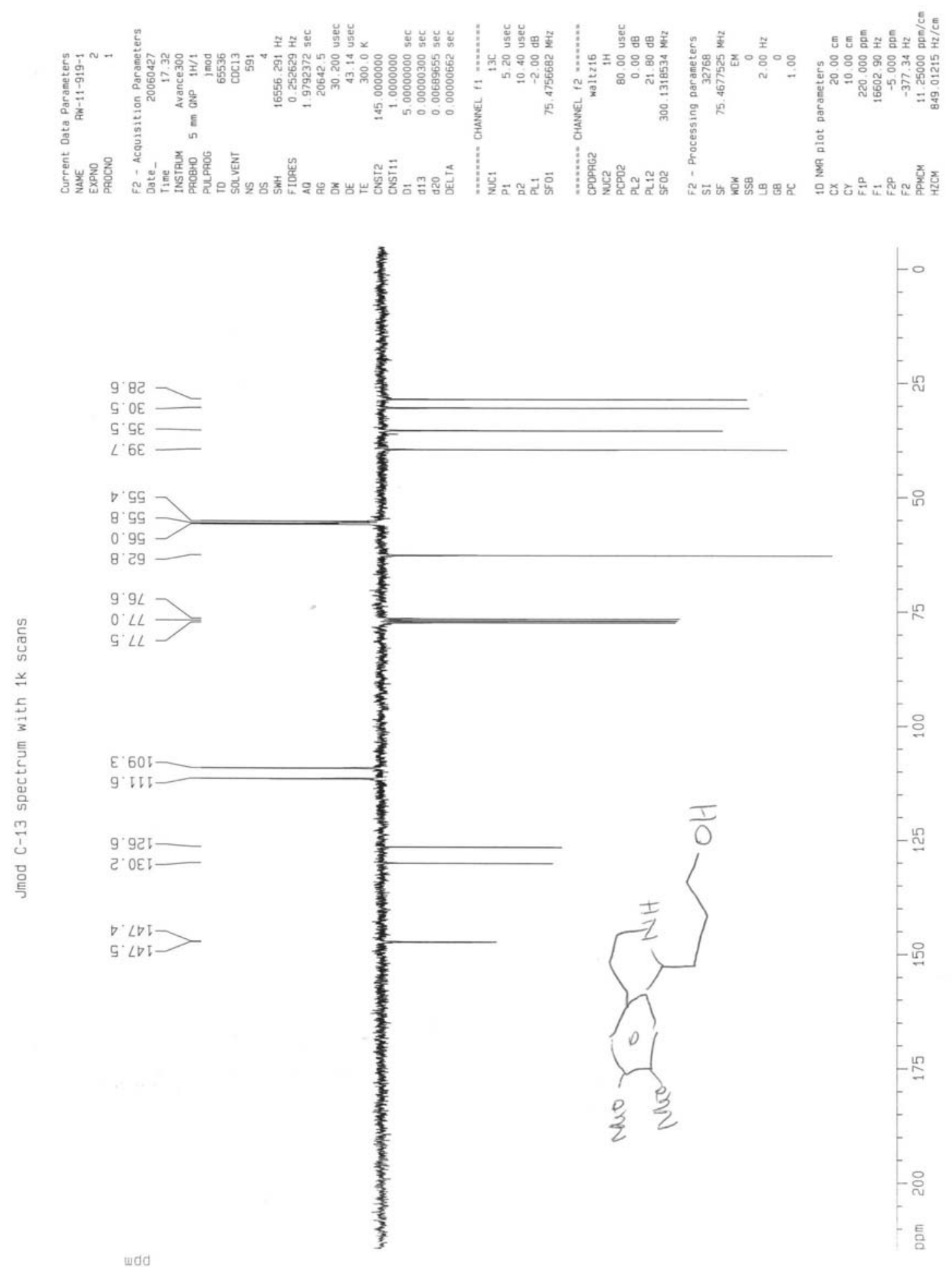


\section{${ }^{1} \mathrm{H}$ NMR spectrum of compound 4 .}
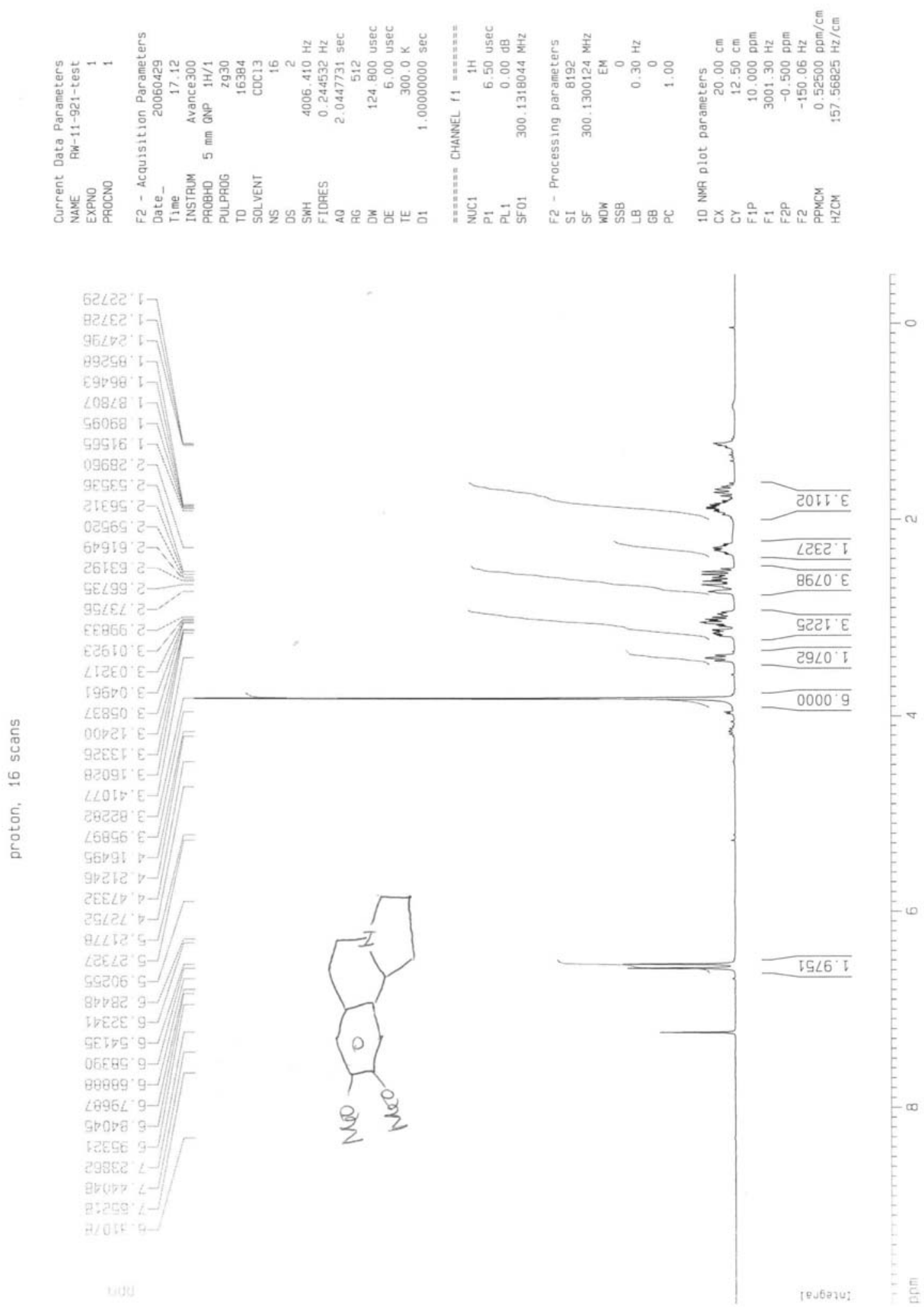


\section{${ }^{13} \mathrm{C}$ NMR spectrum of compound 4.}
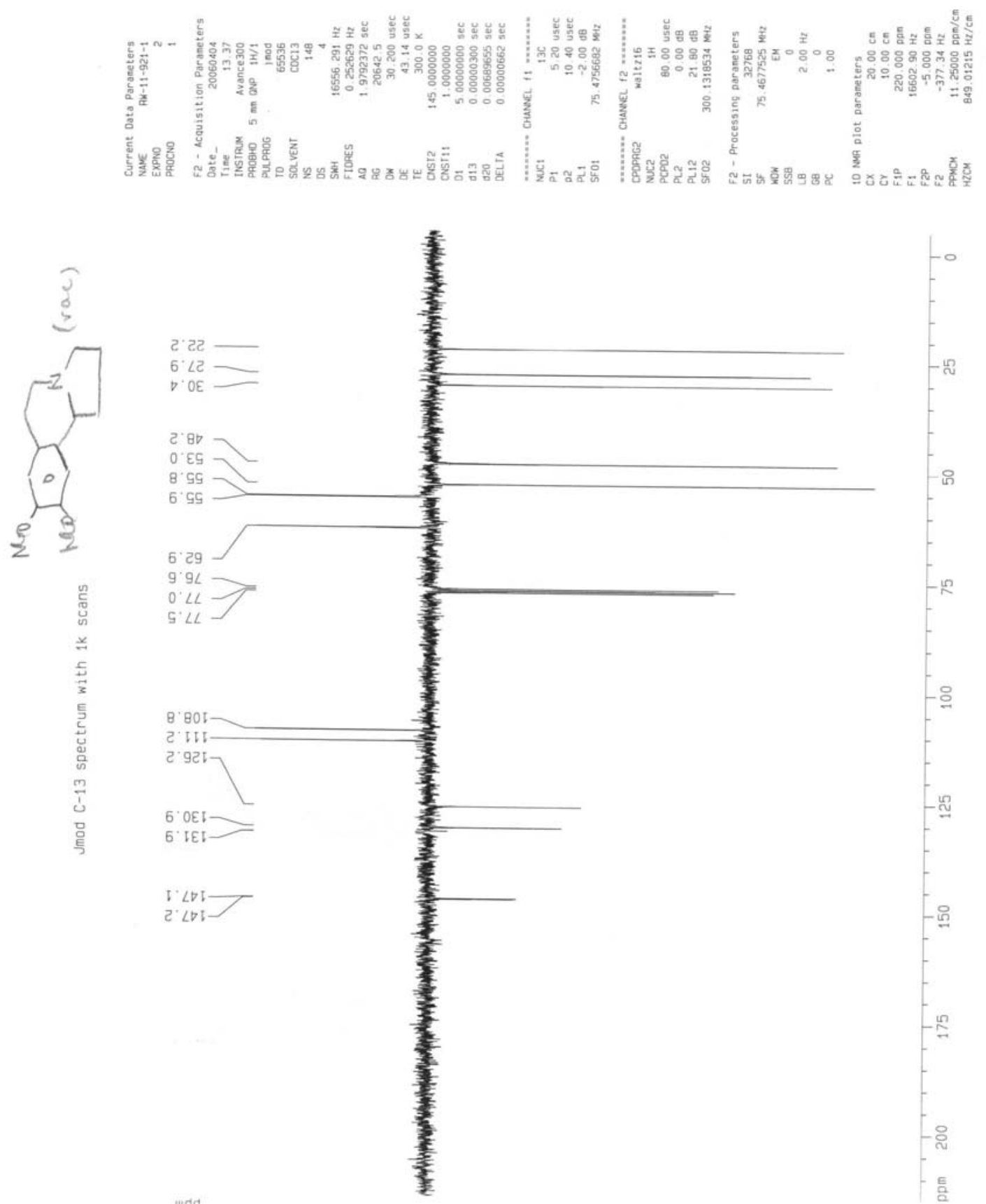


\section{${ }^{1} \mathrm{H}$ NMR spectrum of compound 5 [(R)-Coniine].}
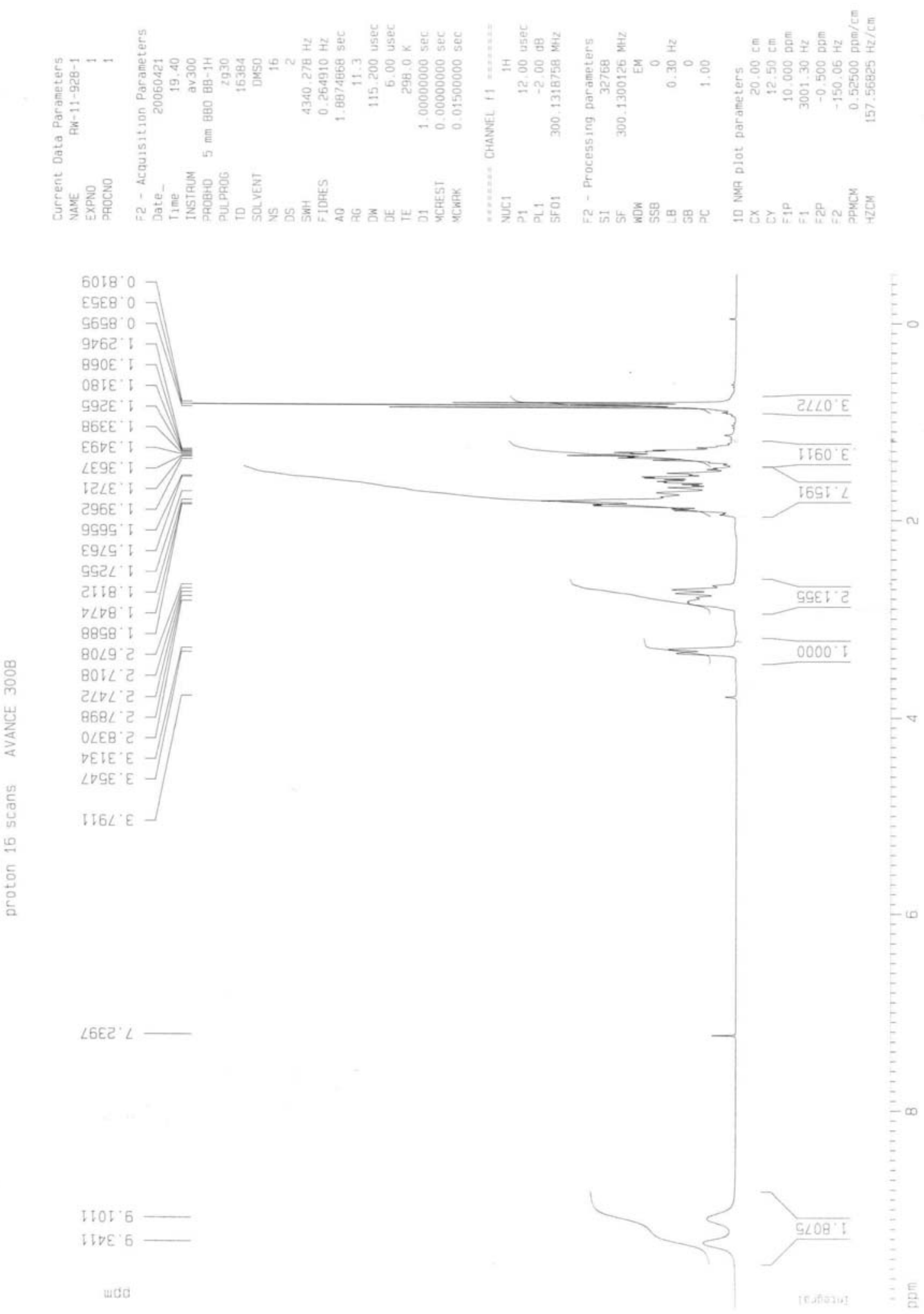


\section{${ }^{13} \mathrm{C}$ NMR spectrum of compound 5.}
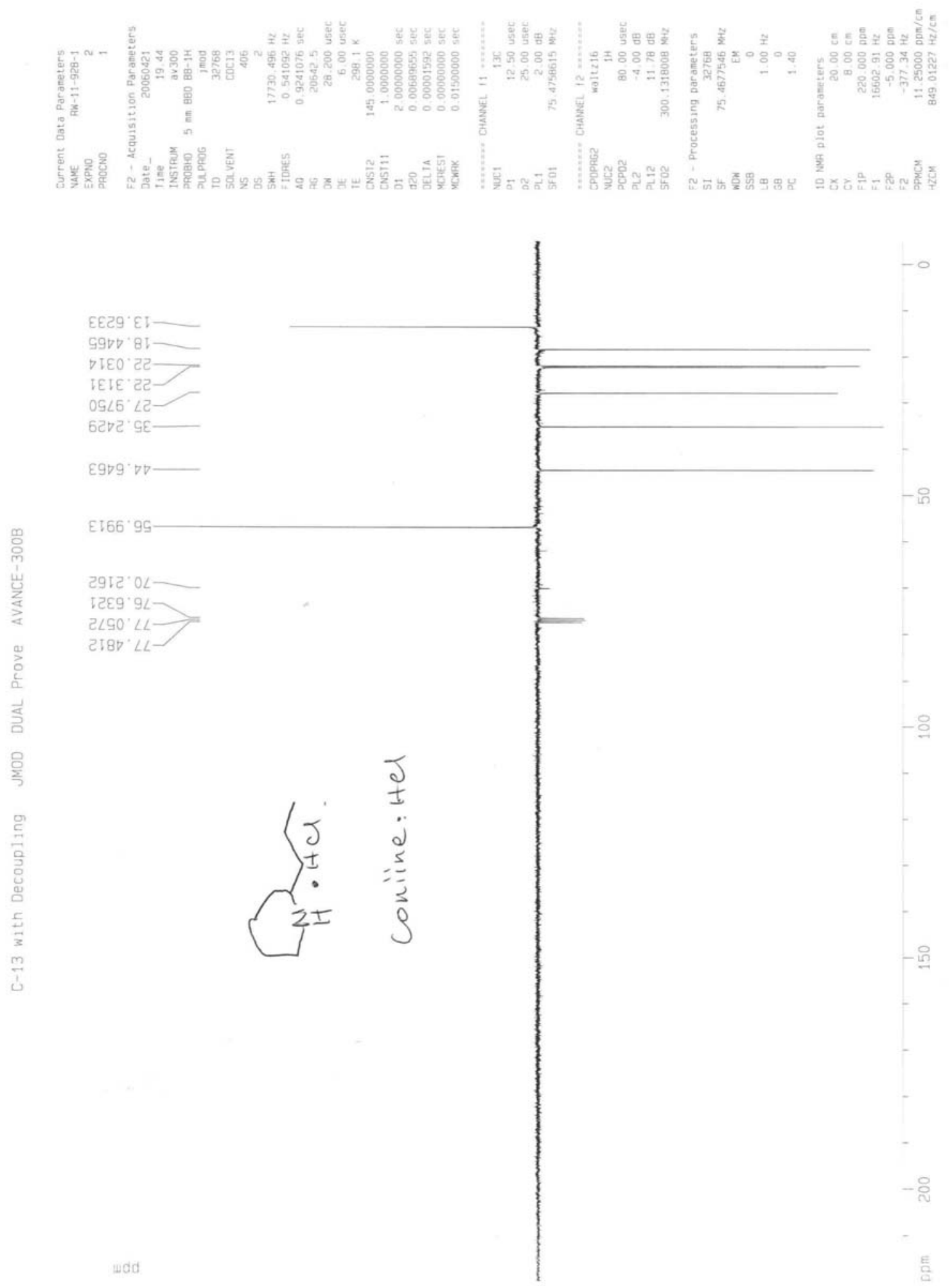


\section{${ }^{1} \mathrm{H}$ NMR spectrum of compound 7.}
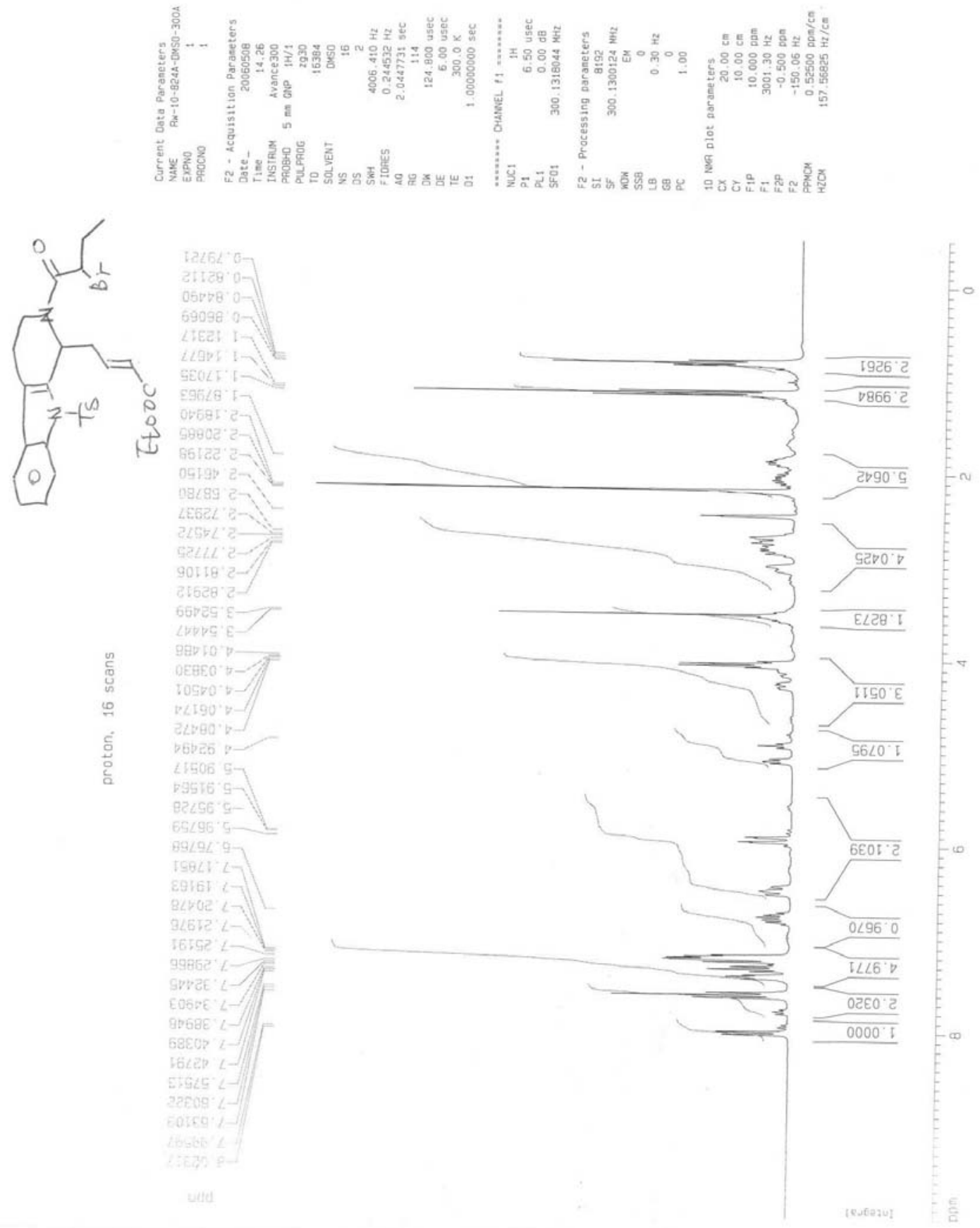


\section{${ }^{13} \mathrm{C}$ NMR spectrum of compound 7.}
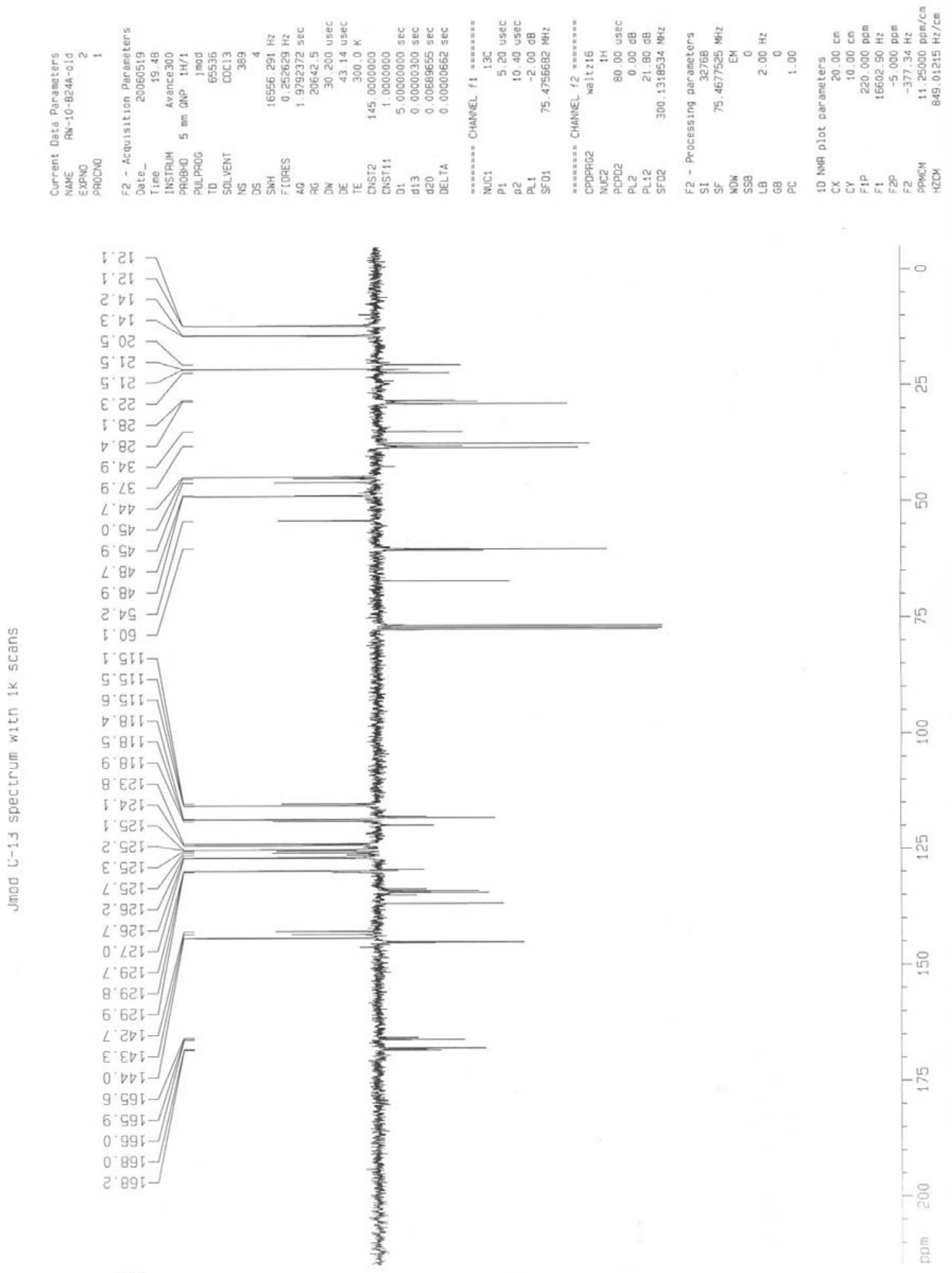


\section{${ }^{1} \mathrm{H}$ NMR spectrum of compound 8.}
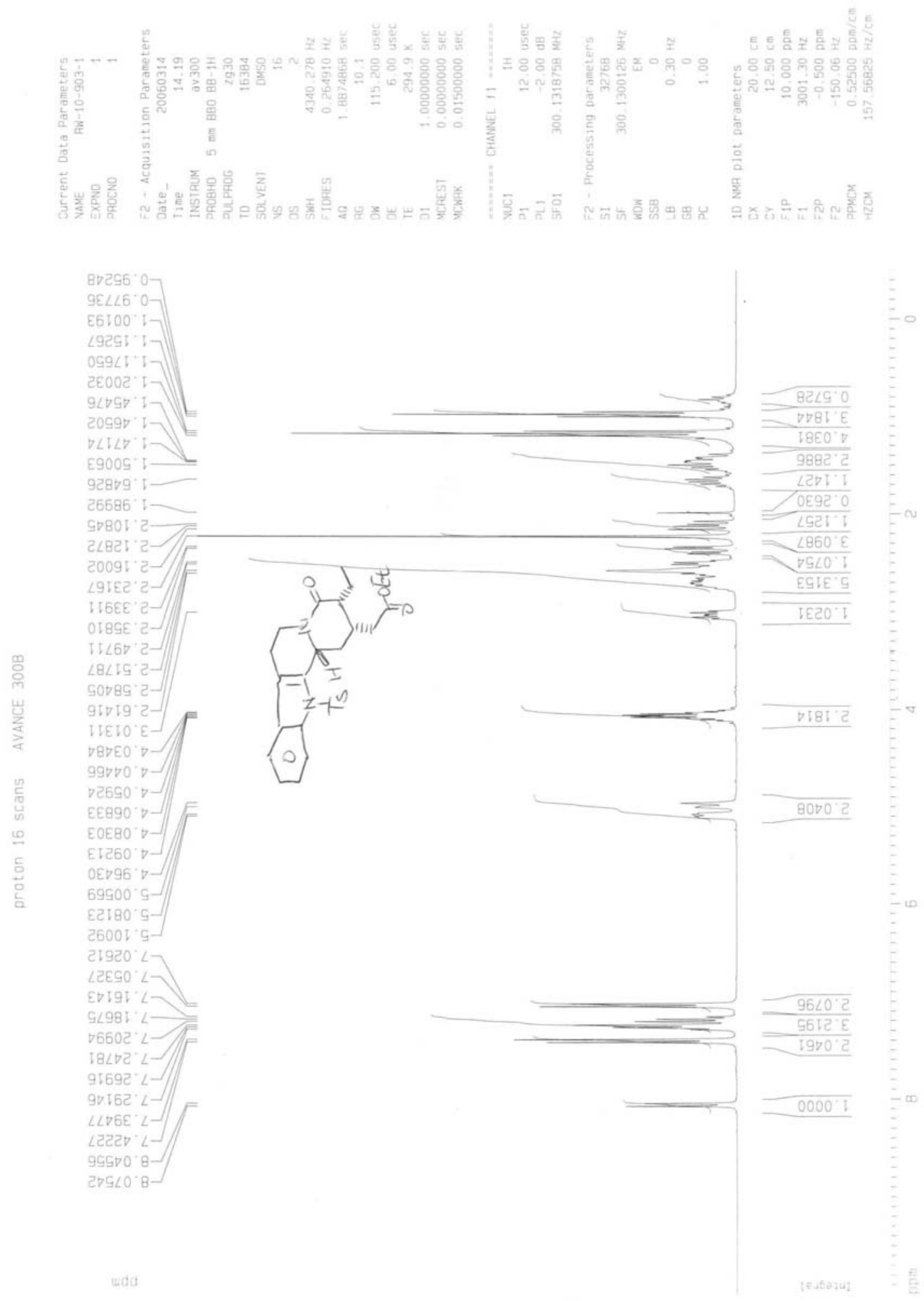
${ }^{13}$ C NMR spectrum of compound 8.

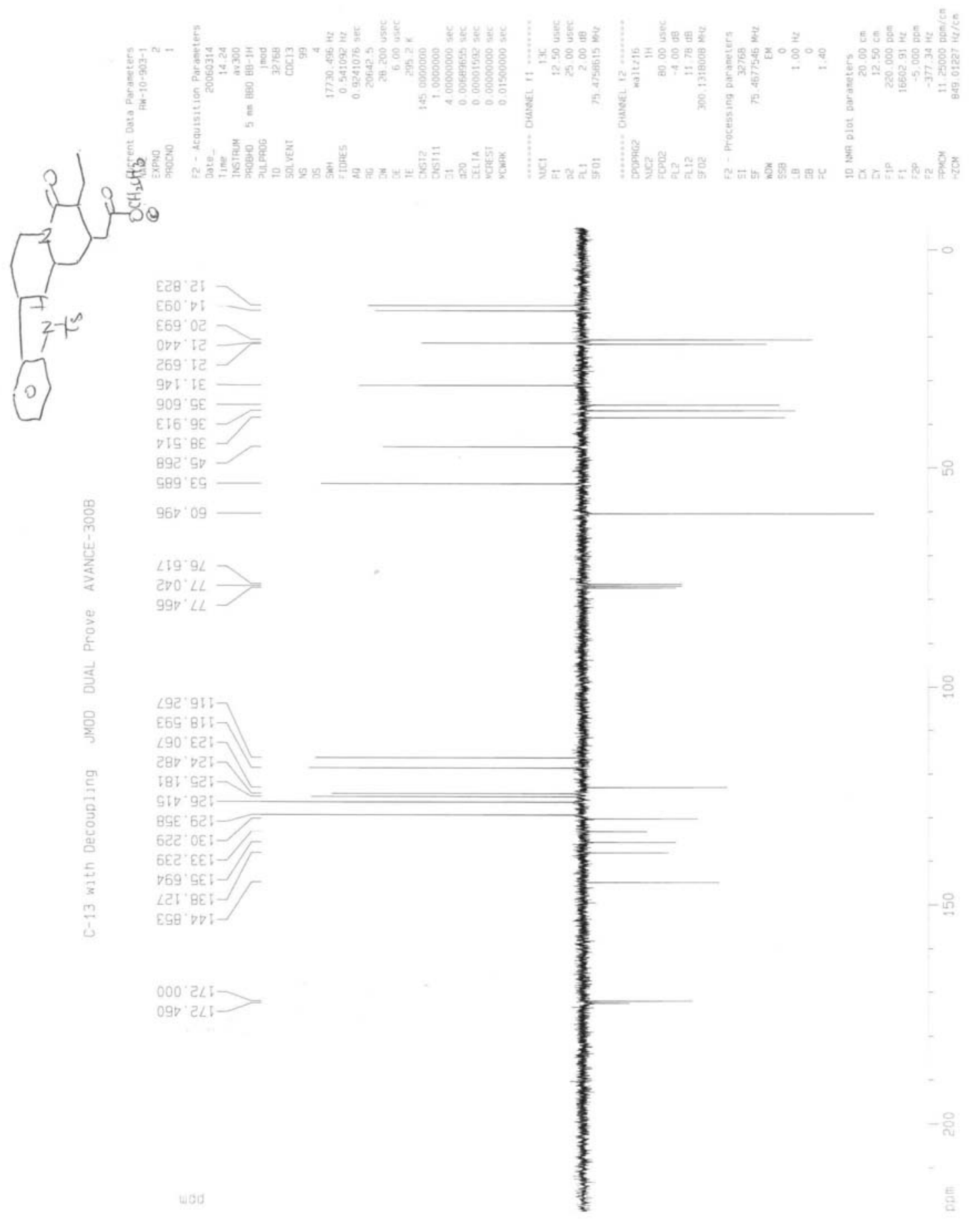


${ }^{1} \mathrm{H}$ NMR spectrum of compound 9 (ent-corynantheidol).
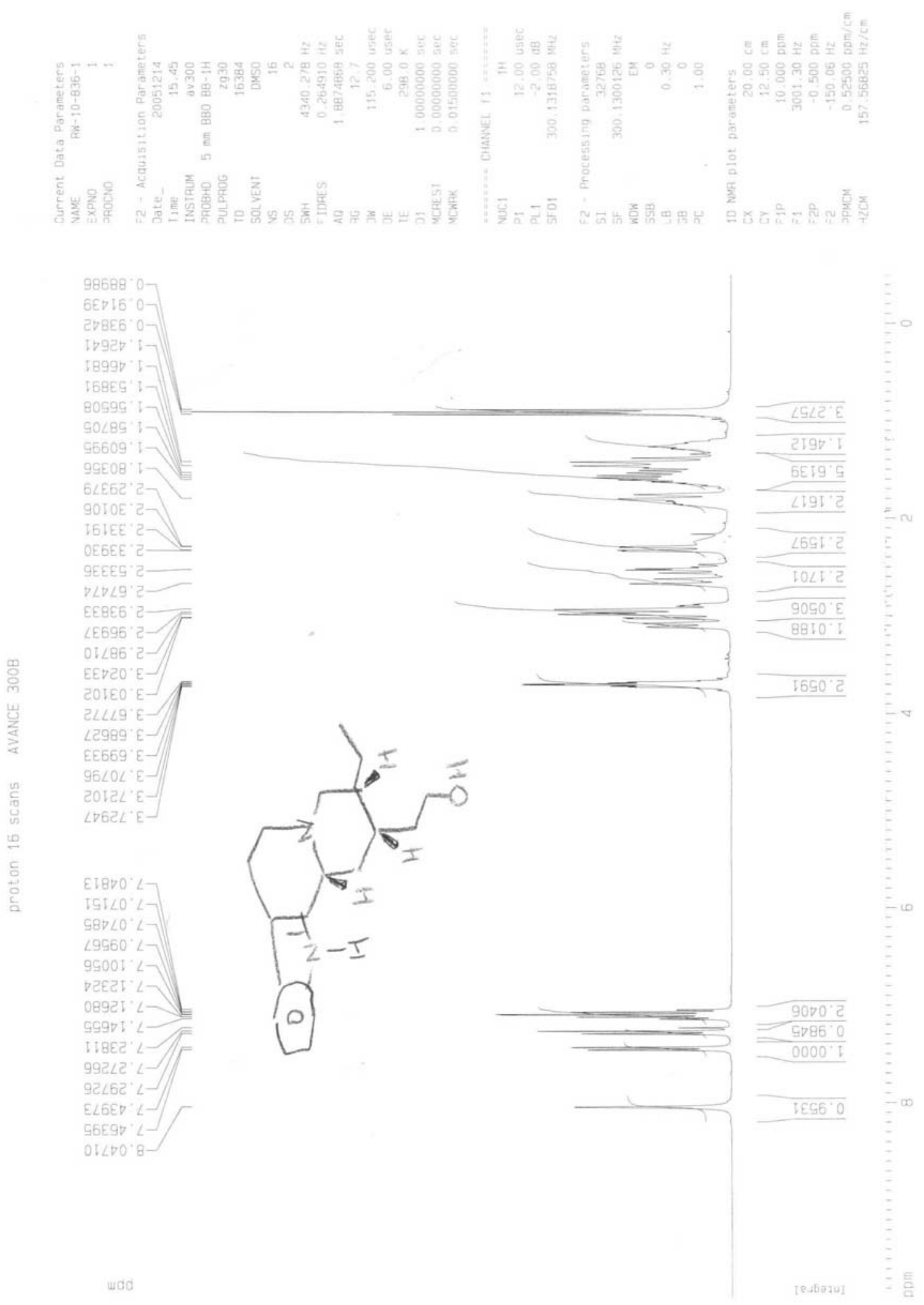
${ }^{13} \mathrm{C}$ NMR spectrum of compound 9 (ent-corynantheidol).
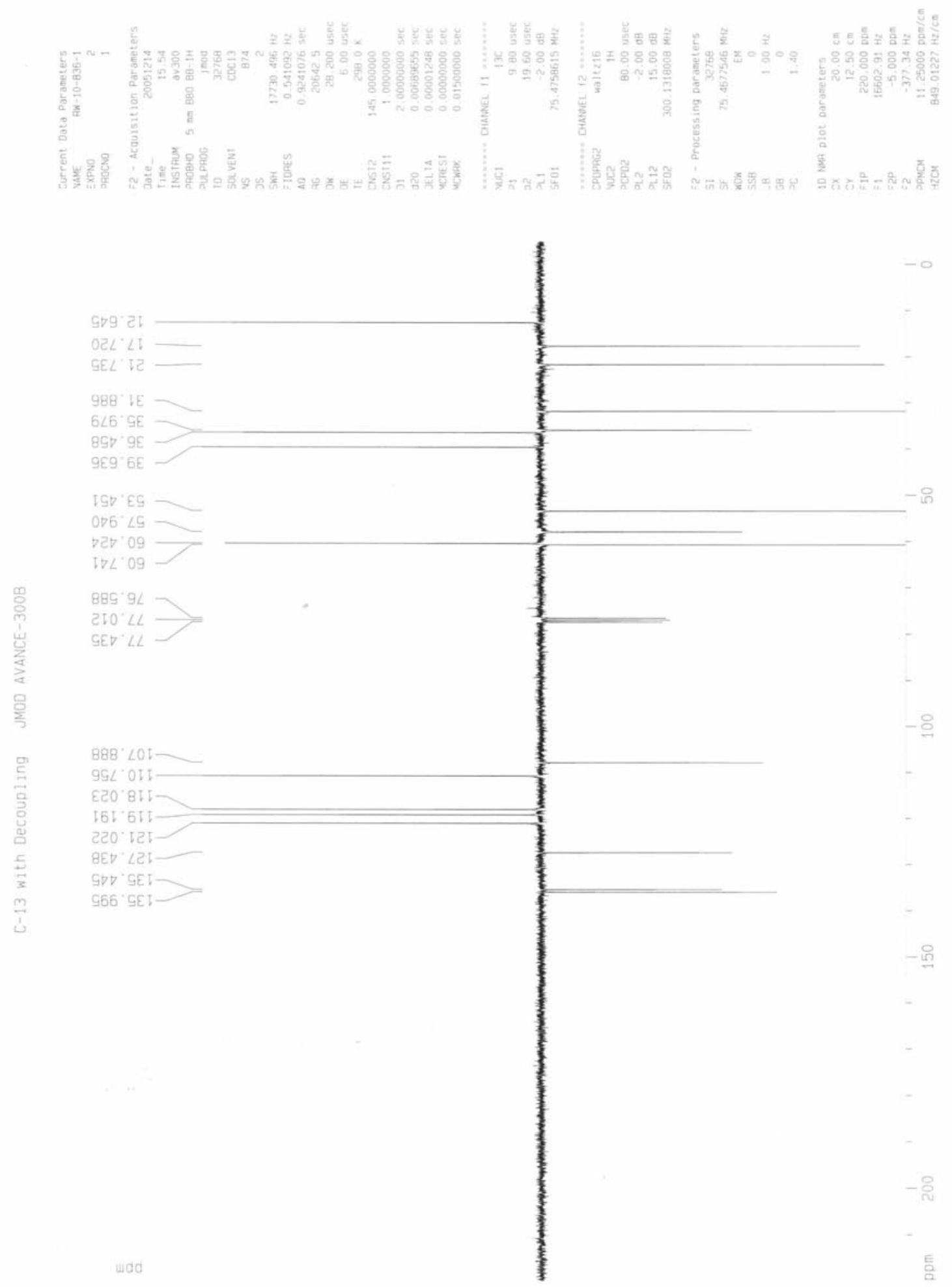